
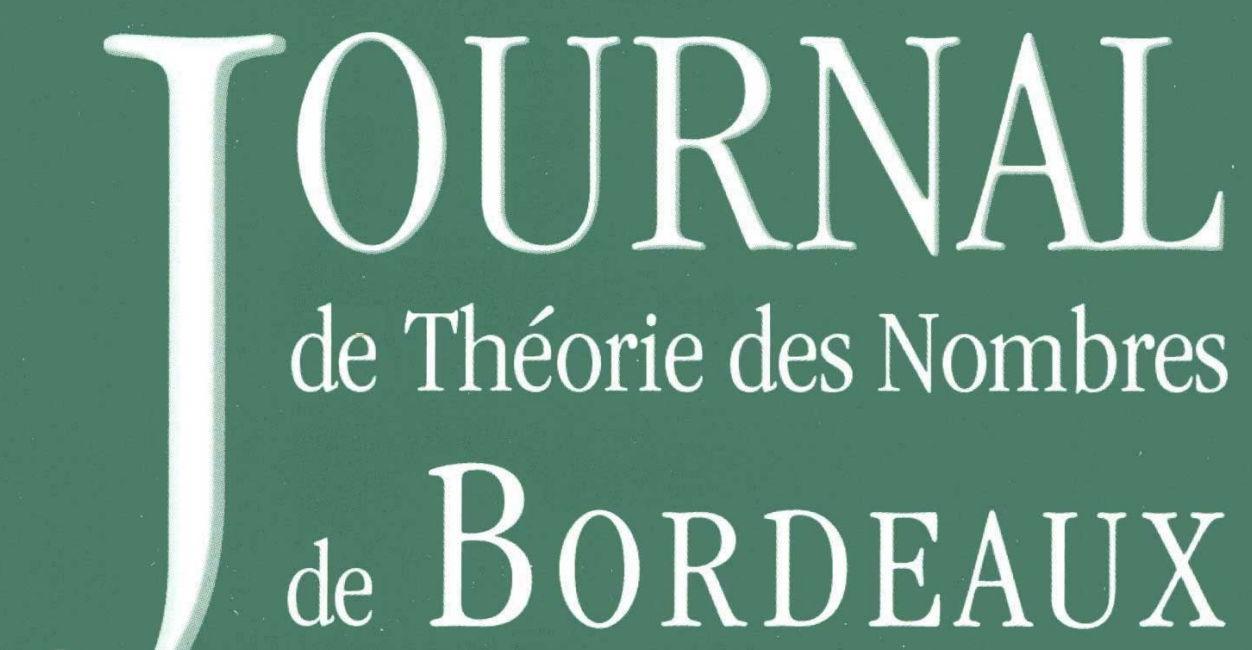

anciennement Séminaire de Théorie des Nombres de Bordeaux

\title{
Shōta INOUE
}

\section{Some explicit formulas for partial sums of Möbius functions}

Tome 33, no 2 (2021), p. 273-315.

<http://jtnb.centre-mersenne.org/item?id=JTNB_2021 33_2_273_0>

(C) Société Arithmétique de Bordeaux, 2021, tous droits réservés. L'accès aux articles de la revue «Journal de Théorie des Nombres de Bordeaux » (http://jtnb.centre-mersenne.org/), implique l'accord avec les conditions générales d'utilisation (http://jtnb. centre-mersenne.org/legal/). Toute reproduction en tout ou partie de cet article sous quelque forme que ce soit pour tout usage autre que l'utilisation à fin strictement personnelle du copiste est constitutive d'une infraction pénale. Toute copie ou impression de ce fichier doit contenir la présente mention de copyright.

\section{cedram}

Article mis en ligne dans le cadre du

Centre de diffusion des revues académiques de mathématiques

http://www.centre-mersenne.org/ 


\title{
Some explicit formulas for partial sums of Möbius functions
}

\author{
par SHŌTA INOUE
}

\begin{abstract}
RÉSUMÉ. Le but de cet article est de donner quelques formules explicites faisant intervenir des fonctions de Möbius. De telles formules explicites peuvent être prouvées sous l'hypothèse de Riemann généralisée, mais dans cet article, nous donnons des preuves inconditionnelles. Concrètement, nous prouvons des formules explicites pour des sommes partielles de la fonction de Möbius dans les progressions arithmétiques et pour des sommes partielles des fonctions de Möbius des corps de nombres abéliens. De plus, pour obtenir ces formules explicites, nous étudions un produit eulérien fini provenant d'une relation entre les caractères primitifs et non primitifs.
\end{abstract}

Abstract. The purpose of this paper is to give some explicit formulas involving Möbius functions. Such explicit formulas may be known under the generalized Riemann Hypothesis, but unconditional in this paper. Concretely, we prove explicit formulas of partial sums of the Möbius function in arithmetic progressions and partial sums of the Möbius functions on an Abelian number field. In addition, to obtain these explicit formulas, we study a certain finite Euler product appearing from a relation between primitive characters and imprimitive characters.

\section{Introduction}

The classical explicit formula

$$
M^{*}(x)=\lim _{\nu \rightarrow \infty} \sum_{|\gamma|<T_{\nu}} \frac{x^{\rho}}{\zeta^{\prime}(\rho) \rho}-2+\sum_{n=1}^{\infty} \frac{(-1)^{n-1}(2 \pi / x)^{2 n}}{(2 n) ! n \zeta(2 n+1)}
$$

was shown by Titchmarsh [18] under the assumption of the Riemann Hypothesis and the simplicity of zeros of the Riemann zeta-function $\zeta(s)$. Here, the function $M^{*}(x)$ is defined by

$$
M^{*}(x)=\sum_{n \leq x}^{\prime} \mu(n)
$$

Manuscrit reçu le 17 août 2019, révisé le 11 mars 2021, accepté le 5 juin 2021.

2020 Mathematics Subject Classification. 11A25, 11R42.

Mots-clefs. Möbius Function, Dirichlet L-Functions, Dedekind Zeta-Functions, Gonek-Hejhal Conjecture, Linear Independence Conjecture. 
$\mu(n)$ is the Möbius function, $\sum^{\prime}$ indicates that if $x$ is an integer, then the last term is to be counted with weight $1 / 2$, and $\left\{T_{\nu}\right\}_{\nu=1}^{\infty}$ is a certain sequence satisfying $T_{\nu} \in[\nu, \nu+1]$. In addition, Bartz [1] unconditionally proved the explicit formula

$$
\begin{array}{r}
M^{*}(x)=\lim _{\nu \rightarrow \infty} \sum_{|\gamma|<T_{\nu}} \frac{1}{(m(\rho)-1) !} \lim _{s \rightarrow \rho} \frac{\mathrm{d}^{m(\rho)-1}}{\mathrm{~d} s^{m(\rho)-1}}\left((s-\rho)^{m(\rho)} \frac{x^{s}}{\zeta(s) s}\right) \\
-2+\sum_{n=1}^{\infty} \frac{(-1)^{n-1}(2 \pi / x)^{2 n}}{(2 n) ! n \zeta(2 n+1)},
\end{array}
$$

where $\left\{T_{\nu}\right\}_{\nu=1}^{\infty}$ is a certain sequence satisfying $T_{\nu} \in[\nu, 2 \nu]$. We in this paper give a generalization of this explicit formula to

$$
M^{*}(x ; q, a)=\sum_{\substack{n \leq x \\ n \equiv a \bmod q}}^{\prime} \mu(n),
$$

which is the summatory function of the Möbius function in arithmetic progressions with $(a, q)=1$. To state our main theorem, we define the function $L_{-1}(s ; q, a)$ by

$$
L_{-1}(s ; q, a):=\frac{1}{\varphi(q)} \sum_{\chi \bmod q} \bar{\chi}(a) L(s, \chi)^{-1} .
$$

Here, the sum runs over all Dirichlet characters modulo $q$, and $\varphi(n)$ is the Euler totient function. The following theorem is a generalization of Bartz's formula and a consequence of the main theorem in this paper.

Theorem 1. Let $a, q \in \mathbb{Z}_{>0}$ with $(a, q)=1$. Then, there exist some numbers $T_{\nu} \in[T, 2 T], T_{*} \in\left[T_{\nu}, T_{\nu}+1\right]$ such that

$$
\begin{aligned}
& \text { (1.4) } M^{*}(x ; q, a) \\
& =\lim _{T \rightarrow+\infty}\left\{\sum_{|\gamma|<T_{\nu}} \frac{1}{(m(\rho)-1) !} \lim _{s \rightarrow \rho} \frac{\mathrm{d}^{m(\rho)-1}}{\mathrm{~d} s^{m(\rho)-1}}\left((s-\rho)^{m(\rho)} L_{-1}(s ; q, a) \frac{x^{s}}{s}\right)\right. \\
& \left.\quad+\sum_{|\eta|<T_{*}} \operatorname{Res}_{s=i \eta}\left(L_{-1}(s ; q, a) \frac{x^{s}}{s}\right)\right\}+\sum_{l=1}^{\infty} \operatorname{Res}_{s=-l}\left(L_{-1}(s ; q, a) \frac{x^{s}}{s}\right) .
\end{aligned}
$$

Here, the first sum runs over non-trivial zeros $\rho=\beta+i \gamma$ of all Dirichlet $L$-functions modulo $q, m(\rho)$ indicates the multiplicity of $\rho$, and the second sum runs over zeros in of all imprimitive Dirichlet L-functions modulo $q$ on the imaginary axis.

The proof of this theorem requires some estimates of Dirichlet $L$-function in certain domains. Hence, we give a new estimate for Dirichlet $L$-functions. 
The estimate is a generalization of the result due to Ramachandra and Sankaranarayanan [16, Theorem 2].

Unfortunately, it is at present difficult to apply these explicit formulas to some problems because there are some inconvenient points. For example, the main terms of formulas (1.2) and (1.4) are more complicated for higher multiplicity zeros, and it is difficult to understand the behavior of multiplicity of nontrivial zeros. We do not know even the boundedness of multiplicity at present. Even if we assume the simplicity of zeros, there is another problem, that is the behavior of $\zeta^{\prime}(\rho)$. This is also difficult because this problem is related to the detailed information on the gaps between zeros of the Riemann zeta-function. For this problem, Gonek [5] and Hejhal [7] independently proposed the following conjecture.

Conjecture (The Gonek-Hejhal Conjecture). Assume the simple zero conjecture for the Riemann zeta-function. For $\lambda>-\frac{3}{2}$,

$$
\sum_{0<\gamma<T}\left|\zeta^{\prime}(\rho)\right|^{2 \lambda} \asymp T(\log T)^{(\lambda+1)^{2}} .
$$

Applying this conjecture and the Riemann Hypothesis to a certain truncated form of (1.1), $\mathrm{Ng}$ [15] proved the following sharp estimate

$$
M^{*}(x) \ll x^{1 / 2}(\log x)^{5 / 4} .
$$

This estimate is stronger than the result

$$
M^{*}(x) \ll x^{1 / 2} \exp \left((\log x)^{1 / 2}(\log \log x)^{14}\right),
$$

which Soundararajan [17] showed under the Riemann Hypothesis alone. From the above background, it can be seen that the truncated explicit formulas are important to obtain the exact upper bound for the summatory functions of Möbius functions.

1.1. Outline of this paper. The rest of this paper is organized as follows: In Section 2 we state our main theorem and its application. In Section 3 we describe some auxiliary results needed in the proof of our main theorem. In Section 4 we prove some estimates of Dirichlet $L$-functions associated with primitive characters in the critical strip. In Section 5 we show some properties of the finite Euler product appearing in the expression of Dirichlet $L$-functions attached to imprimitive characters. In Section 6 we give a proof of some explicit formulas involving the main theorem. In Section 7 we evaluate the contribution from zeros of Dirichlet $L$-functions on the imaginary axis and trivial zeros. In Section 8 we prove an approximate formula for a sum of $1 / L^{\prime}(\rho, \chi)$. In Section 9 we describe results for certain number fields, and prove those in Section 10. 
Acknowledgments. The author expresses his gratitude to Professor Kohji Matsumoto for his helpful comments. The author would like to thank the referees for their careful reviews and valuable comments.

\section{Statement of the main theorem and its application}

The present paper gives some truncated explicit formulas, which generalize the truncated form of (1.2). Our main result is an explicit formula for $M^{*}(x ; q, a)$, which is a truncated one of Theorem 1 . Define $\langle x\rangle$ by the distance from $x$ to the nearest square-free integers coprime to $q$, other than $x$ itself. Then we show the following truncated formula for $M^{*}(x ; q, a)$.

Theorem 2. Let $x>0, T \geq \max \left\{T_{0}, \exp \left(q^{1 / 3}\right), 2 / x\right\}$ with $T_{0}$ a sufficiently large absolute constant, $a, q \in \mathbb{Z}_{>0}$ with $(a, q)=1$. Then, there exist some numbers $T_{\nu} \in[T, 2 T], T^{*} \in\left[T_{\nu}, T_{\nu}+1\right]$ such that

$$
\begin{aligned}
& M^{*}(x ; q, a) \\
& =\sum_{|\gamma|<T_{\nu}} \frac{1}{(m(\rho)-1) !} \lim _{s \rightarrow \rho} \frac{\mathrm{d}^{m(\rho)-1}}{\mathrm{~d} s^{m(\rho)-1}}\left((s-\rho)^{m(\rho)} L_{-1}(s ; q, a) \frac{x^{s}}{s}\right) \\
& \quad+\sum_{|\eta|<T_{*}} \operatorname{Res}_{s=i \eta}\left(L_{-1}(s ; q, a) \frac{x^{s}}{s}\right)+\sum_{l=1}^{\infty} \operatorname{Res}_{s=-l}\left(L_{-1}(s ; q, a) \frac{x^{s}}{s}\right)+R .
\end{aligned}
$$

Here, the first sum runs over non-trivial zeros $\rho=\beta+i \gamma$ of all Dirichlet $L$-functions modulo $q, m(\rho)$ indicates the multiplicity of $\rho$, and the second sum runs over zeros in of all imprimitive Dirichlet $L$-functions modulo $q$ on the imaginary axis. The error term $R$ satisfies

$$
R \ll \frac{x}{T}\left(\log (x+3)+\exp \left(C(\log \log T)^{2}\right)\right)+\min \left\{1, \frac{x}{T\langle x\rangle}\right\},
$$

where this implicit constant is absolute, and $C$ is an absolute positive constant.

We evaluate the contribution from the residues of trivial zeros and of zeros on the imaginary axis in Section 7 .

Now we consider the upper bound of $M^{*}(x ; q, a)$ under some assumptions. One of those assumptions is a generalization of the Gonek-Hejhal Conjecture, which is the following assertion.

Conjecture 1 (The Generalized Gonek-Hejhal Conjecture for Dirichlet $L$-functions). Assume that the all zeros of all Dirichlet $L$-functions are simple except for the zero at $s=\frac{1}{2}$. For any number $T \geq 3$,

$$
J_{\lambda, \chi}(T):=\sum_{0<|\gamma| \leq T}\left|L^{\prime}(\rho, \chi)\right|^{2 \lambda} \asymp_{\lambda, q} T(\log T)^{(\lambda+1)^{2}}
$$

hold for $\lambda>-\frac{3}{2}$, where $q$ is the modulus of $\chi$. 
Remark 1. This conjecture is just a generalization of the case of the Riemann zeta-function, and the author does not know any further evidence.

Now we obtain the following corollary under the generalized Riemann Hypothesis and Conjecture 1.

Theorem 3. Let $a, q \in \mathbb{Z}_{>0}$ with $(a, q)=1$. Assume that the generalized Riemann Hypothesis for all Dirichlet L-functions modulo $q$ is true, the all zeros of all Dirichlet L-functions are simple except for the zero at $s=\frac{1}{2}$, and that $J_{-1 / 2, \chi}(T) \ll_{q} T(\log T)^{1 / 4}$. Then, we have

$$
M^{*}(x ; q, a) \ll_{q} x^{1 / 2}\left((\log x)^{5 / 4}+(\log x)^{m(q)-1}\right),
$$

where $m(q)$ is the maximum in multiplicities of the zero of Dirichlet $L$ functions modulo $q$ at $s=\frac{1}{2}$.

Presently, the best upper bound of $M^{*}(x ; q, a)$ assuming only the generalized Riemann Hypothesis is the following estimate

$$
M^{*}(x ; q, a) \ll_{\varepsilon} x^{1 / 2} \exp \left((\log (x / d))^{1 / 2}(\log \log (x / d))^{3+\varepsilon}\right),
$$

which was shown by Ye [19]. Here the number $d$ indicates $\operatorname{gcd}(a, q)$. Estimate (2.4) is a result under the assumption of the generalized Gonek-Hejhal Conjecture in addition to the generalized Riemann Hypothesis. However, thanks to explicit formula (2.1) and estimate (2.4), we can grasp the more accurate behavior of $M^{*}(x ; q, a)$.

\section{Auxiliary results and their applications}

The function $M^{*}(x ; q, a)$ can be expressed by

$$
M^{*}(x ; q, a)=\frac{1}{\varphi(q)} \sum_{\chi \bmod q} \bar{\chi}(a) \sum_{n \leq x}^{\prime} \chi(n) \mu(n)
$$

from the orthogonality of characters. Here the first sum runs over all Dirichlet characters modulo $q$, and $\varphi$ is the Euler totient function. Therefore, as the first step, we show the explicit formulas for the summatory function

$$
M^{*}(x, \chi)=\sum_{n \leq x}^{\prime} \chi(n) \mu(n) .
$$

3.1. Explicit formulas for $M^{*}(x, \chi)$. We give two explicit formulas for $M^{*}(x, \chi)$ separately in the cases of primitive and imprimitive characters. 
Theorem 4. Let $x>0, q \geq 2, T \geq \max \left\{T_{0}, \exp \left(q^{1 / 3}\right), 2 / x\right\}$ with $T_{0}$ a sufficiently large absolute constant. Then, uniformly for any primitive Dirichlet character $\chi$ modulo $d$ with $d \leq q$, there exists a number $T_{\nu} \in$ $[T, 2 T]$ such that

$$
\begin{array}{r}
M^{*}(x, \chi)=\sum_{|\gamma|<T_{\nu}} \frac{1}{(m(\rho)-1) !} \lim _{s \rightarrow \rho} \frac{\mathrm{d}^{m(\rho)-1}}{\mathrm{~d} s^{m(\rho)-1}}\left(\frac{(s-\rho)^{m(\rho)}}{L(s, \chi)} \frac{x^{s}}{s}\right) \\
+\operatorname{Res}_{s=0}\left(\frac{x^{s}}{L(s, \chi) s}\right)+\sum_{l=1}^{\infty} \operatorname{Res}_{s=-l}\left(\frac{x^{s}}{L(s, \chi) s}\right)+R,
\end{array}
$$

where $R$ is the error term satisfying the estimate (2.2). The first sum on the right hand side runs over nontrivial zeros $\rho=\beta+i \gamma$ of $L(s, \chi)$.

This explicit formula is the case of primitive characters. On the other hand, for our purpose, we need an analogue of Theorem 4 for imprimitive characters. Here we can associate an imprimitive character $\chi$ with a primitive character $\chi^{*}$ inducing $\chi$ by the formula

$$
L(s, \chi)=L\left(s, \chi^{*}\right) \prod_{p \mid q}\left(1-\frac{\chi^{*}(p)}{p^{s}}\right) .
$$

Here we put

$$
F_{q, \chi^{*}}(s):=\prod_{p \mid q}\left(1-\frac{\chi^{*}(p)}{p^{s}}\right) .
$$

In the following, we consider the case $F_{q, \chi^{*}} \not \equiv 1$. Then this function $F_{q, \chi^{*}}$ has zeros only on the imaginary axis. In addition, from the uniqueness of the prime factorization, we can see that all zeros of $F_{q, \chi^{*}}$ are simple except the zero at $s=0$. Now, by studying $F_{q, \chi^{*}}$, we obtain an explicit formula for imprimitive characters as the following theorem.

Theorem 5. Let $x>0, q \geq 2, T \geq \max \left\{T_{0}, \exp \left(q^{1 / 3}\right), 2 / x\right\}$ with $T_{0}$ a sufficiently large absolute constant, $\chi$ be an imprimitive Dirichlet character modulo $q$. Then, uniformly for any imprimitive Dirichlet character $\chi$ modulo $q$ with $F_{q, \chi^{*}} \not \equiv 1$, there exist some numbers $T_{\nu} \in[T, 2 T]$ and $T_{*} \in\left[T_{\nu}, T_{\nu}+1\right]$ such that

$$
\begin{aligned}
& M^{*}(x, \chi)=\sum_{|\gamma|<T_{\nu}} \frac{1}{(m(\rho)-1) !} \lim _{s \rightarrow \rho} \frac{\mathrm{d}^{m(\rho)-1}}{\mathrm{~d} s^{m(\rho)-1}}\left(\frac{(s-\rho)^{m(\rho)}}{L(s, \chi)} \frac{x^{s}}{s}\right) \\
& +\sum_{|\eta|<T_{*}} \operatorname{Res}_{s=i \eta}\left(\frac{x^{s}}{L(s, \chi) s}\right)+\sum_{l=1}^{\infty} \operatorname{Res}_{s=-l}\left(\frac{x^{s}}{L(s, \chi) s}\right)+R,
\end{aligned}
$$

where the second sum on the right hand side runs over zeros of $L(s, \chi)$ on the imaginary axis, and $R$ is the error term satisfying estimate (2.2). 
From the above two theorems and equation (3.1), we can obtain Theorem 2 .

3.2. Uniform upper bounds on certain horizontal lines. To prove the above theorems, we need some upper bound of $1 / L(s, \chi)$ in certain domains, which is embodied in the following two propositions.

Proposition 1. Let $\alpha \geq 13, T \geq T_{0}(\alpha)>0$, and $1 \leq Q \leq(\log T)^{\alpha / 4}$, where $T_{0}(\alpha)$ is a sufficiently large constant depending only on $\alpha$. Then, we have

$$
\min _{T \leq t \leq 2 T}\left(\max _{\substack{\frac{1}{2} \leq \sigma \leq 2 \\ \chi \in S(Q)}}|L(\sigma+i t, \chi)|^{-1}\right) \leq \exp \left(C \alpha(\log \log (Q T))^{2}\right),
$$

where $C$ is a positive absolute constant, and $S(Q)$ is the set of all primitive Dirichlet characters modulo $q$ with $q \leq Q$.

This proposition holds for primitive Dirichlet characters. On the other hand, we need a similar result for imprimitive Dirichlet characters to prove Theorem 5 . Then we need the upper bound of $1 / F_{q, \chi^{*}}$, which is given by the following proposition. Define $\omega(n)$ by the number of distinct prime factors of $n$, and $\operatorname{rad}(n)=\prod_{p \mid n} p$.

Proposition 2. Let $q \geq 2$ be an integer, $|T| \geq \omega(q), S_{1}(q)$ be a nonempty subset of the set of all imprimitive Dirichlet characters modulo $q$ with $F_{q, \chi^{*}} \not \equiv 1$, and $d$ be the smallest modulus of a primitive character $\chi^{*}$ inducing $\chi$ with $\chi \in S_{1}(q)$. Then we have

$$
\begin{aligned}
& \min _{t \in[T, T+1]}\left(\max _{\substack{|\sigma| \leq h \\
\chi \in S_{1}(q)}}\left|F_{q, \chi^{*}}(\sigma+i t)\right|^{-1}\right) \\
& \leq \exp \left(C \omega\left(q^{\prime} / d^{\prime}\right)\right. \log \left(\# S_{1}(q) \omega\left(q^{\prime} / d^{\prime}\right)+2\right) \\
&\left.\times\left(1+\sqrt{\frac{\log \left(q^{\prime} / d^{\prime}\right) / \omega\left(q^{\prime} / d^{\prime}\right)}{\log \left(\# S_{1}(q) \omega\left(q^{\prime} / d^{\prime}\right)+2\right)}}\right)\right),
\end{aligned}
$$

where $C$ is a sufficiently large positive absolute constant, $q^{\prime}=\operatorname{rad}(q), d^{\prime}=$ $\operatorname{rad}(d)$, and $h \asymp \sqrt{\frac{\omega\left(q^{\prime} / d^{\prime}\right) / \log \left(q^{\prime} / d^{\prime}\right)}{\log \left(\# S_{1}(q) \omega\left(q^{\prime} / d^{\prime}\right)+2\right)}}$.

We are going to prove some properties of $F_{q, \chi^{*}}$ including this proposition in Section 5.

Now, we remark that Proposition 1 is an extension to Dirichlet $L$-functions of the result in the case of the Riemann zeta-function by Ramachandra 
and Sankaranarayanan [16, Theorem 1.2]. This result is useful when we prove some explicit formulas including the above formulas. For example, Kühn, Robles and Roy showed an explicit formula involving the Möbius function and a primitive Dirichlet character under the Riemann Hypothesis and the simple zero conjecture for Dirichlet $L$-functions [9, Theorem 1.1(ii)]. The author expects that it is possible to prove their explicit formula without the Riemann Hypothesis for Dirichlet $L$-functions. In fact, they use the Riemann Hypothesis for Dirichlet $L$-functions only in the proof of their Lemma 2.2, and in this paper, we are going to prove Proposition 1, which is an unconditional alternative of their Lemma 2.2.

One more useful point of Proposition 1 is the uniformity for Dirichlet characters modulo $q$ with $q \leq Q$. From this uniformity, there are some applications. For example, one of the applications is that we can take $T_{\nu}$ not depending on the characters modulo $q$ in Corollary 2. Thanks to this fact, we can apply Proposition 1 to Abelian number fields. We describe this application to number fields in Chapter 9.

3.3. An application to a sum over zeros of $L(s, \chi)$. As another application of Proposition 1, we can prove the following theorem for the sum involving derivative functions. The theorem is a generalization of the result in the case of the Riemann zeta-function by Garaev and Sankaranarayanan [4].

Theorem 6. Let $\chi$ be a primitive Dirichlet character modulo $q$, and assume the simplicity of all complex zeros of $L(s, \chi)$. Then, for $T>\exp \left(q^{1 / 3}\right)$, there exist some $T_{\nu} \in[T, 2 T]$ satisfying

$$
\sum_{0<\gamma<T_{\nu}} \frac{1}{L^{\prime}(\rho, \chi)}=\frac{T_{\nu}}{2 \pi}+O\left(\exp \left(C(\log \log T)^{2}\right)+C(\chi)\right),
$$

where the sum on the left hand side runs over non-trivial zeros $\rho=\beta+i \gamma$ of $L(s, \chi)$, and $C(\chi)$ is a sufficiently large constant depending only on $\chi$. Moreover, for any $T>T_{0}(q)>0$ with a sufficiently large constant $T_{0}(q)$ depending only on $q$, we have

$$
\sum_{0<\gamma \leq T} \frac{1}{\left|L^{\prime}(\rho, \chi)\right|} \gg T
$$

In particular, we also have

$$
\frac{1}{\varphi(q)} \sum_{\chi \bmod } \sum_{q 0<\gamma \leq T} \frac{1}{\left|L^{\prime}(\rho, \chi)\right|} \gg T .
$$

Here we mention an application of estimate (3.4). This result is useful when we consider the exact behavior of some partial sum of some Möbius functions under the Linear Independence Conjecture. Here the Linear Independence Conjecture is the following conjecture. 
Conjecture (Linear Independence Conjecture for Dirichlet $L$-functions (cf. [3])). The positive imaginary parts of the zeros of all Dirichlet $L$ functions are linearly independent over $\mathbb{Q}$.

Now by estimate (3.4), as an extension of Ingham's theorem [8], we can obtain that, for $(a, q)=1$,

$$
\varlimsup_{x \rightarrow \infty} \frac{M^{*}(x ; q, a)}{x^{1 / 2}}= \pm \infty
$$

under the Linear Independence Conjecture for Dirichlet $L$-functions. These proofs are similar to the proof of Corollary 15.7 in [12].

Remark 2. We can generalize Theorem 6 to the statement which is analogous to the Landau-Gonek formula (cf. [2, Proposition 2] and [6, Theorem 1]), i.e. for some $T_{\nu} \in[T, 2 T]$,

$$
\sum_{0<\gamma<T_{\nu}} \frac{x^{\rho}}{L^{\prime}(\rho, \chi)}
$$

are estimated by a little modified asymptotic formula with the original Landau-Gonek formula under the simple zero conjecture for the corresponding function. Moreover, if the Riemann Hypothesis for Dirichlet $L$ functions and $\left|L^{\prime}(\rho, \chi)\right|^{-1} \ll|\rho|^{1-\delta}$ for some fixed constant $\delta>0$ are also true, then we have an analogue of the Landau-Gonek formula for (3.5) for any sufficiently large $T>0$. We only mention this fact here because the author cannot find some useful applications of these consequences.

Here the author raises the following conjecture suggested by the above results.

Conjecture 2. Let $\chi$ be a primitive Dirichlet character, and $K$ be an Abelian number field. Then

$$
\sum_{0<\gamma \leq T} \frac{1}{L^{\prime}(\rho, \chi)} \sim \frac{T}{2 \pi} \quad(T \rightarrow \infty) .
$$

We can prove this conjecture in the case of the Riemann zeta-function under some known conjectures that are the Riemann Hypothesis, the simple zero conjecture and the conjectural estimate $\left|\zeta^{\prime}(\rho)\right|^{-1} \ll|\rho|^{1 / 3+\varepsilon}$. In fact, we can obtain the following asymptotic formula

$$
\sum_{0<\gamma \leq T} \frac{1}{\zeta^{\prime}(\rho)}=\frac{T}{2 \pi}+O_{\varepsilon}\left(T^{1 / 3+\varepsilon}\right)
$$

under these conjectures. The present paper does not give the proof of this estimate because it is almost similar to the proof of Theorem 15.6 in [12]. 


\section{On estimates of Dirichlet $L$-functions in certain domains}

In this section, we are going to show some estimates of Dirichlet $L$ functions including Proposition 1. Firstly, we refer to an important result on the zero density theorem for Dirichlet $L$-functions by Montgomery.

Lemma 1 ([10, Theorem 1]). Let $S(Q)$ denote the set of all primitive Dirichlet characters modulo $q$ with $q \leq Q$. For $Q \geq 1, T \geq 2$, and $\frac{1}{2} \leq \sigma \leq$ 1, we have

$$
\sum_{\chi \in S(Q)} N_{\chi}(\sigma, T) \ll\left(Q^{2} T\right)^{\frac{3(1-\sigma)}{2-\sigma}}(\log (Q T))^{13},
$$

where $N_{\chi}(\sigma, T)$ is the number of zeros $\rho$ of $L(s, \chi)$ with $\operatorname{Re}(\rho) \geq \sigma$ and $|\operatorname{Im}(\rho)| \leq T$.

By using this lemma, we show the following proposition.

Proposition 3. Let $\alpha \geq 13, T \geq T_{0}(\alpha)>0$ with $T_{0}(\alpha)$ a sufficiently large number depending only on $\alpha$, and $1 \leq Q \leq(\log T)^{\alpha / 4}$. Then there exists a closed interval $J_{0}$ of length $(\log (Q T))^{\alpha / 3}$ contained in $[T, 2 T]$ such that

$$
\max _{\substack{\sigma \geq 1 / 2+14 \alpha r \\ t \in J_{0}, \chi \in S(Q)}}|\log L(\sigma+i t, \chi)| \ll \alpha \log \log (Q T),
$$

where $r=(\log \log (Q T))^{2}\left(\log \left(Q^{2} T\right)\right)^{-1}$.

Proof. Let $D=\frac{\alpha}{3}$ and $I_{j}=\left[T+2(j-1)(\log (Q T))^{D}, T+2 j(\log (Q T))^{D}\right)$. By Lemma 1, if $\sigma \geq \frac{1}{2}+\alpha(\log \log (Q T))\left(\log \left(Q^{2} T\right)\right)^{-1}$, then

$$
\sum_{\chi \in S(Q)} N_{\chi}(\sigma, 2 T)-\sum_{\chi \in S(Q)} N_{\chi}(\sigma, T) \leq C Q^{2} T(\log (Q T))^{-\alpha} .
$$

Here $C$ is a sufficiently large absolute constant. Now we consider the disjoint rectangles

$$
(\sigma, t) \in R_{j}=\left[\frac{1}{2}+\alpha(\log \log (Q T))\left(\log \left(Q^{2} T\right)\right)^{-1}, 2\right] \times I_{j},
$$

which we may regard as subsets in the complex plane. The number of these rectangles is $N=\left[\frac{1}{2} T(\log (Q T))^{-D}\right]$. By inequality (4.1), if $Q \leq(\log T)^{\alpha / 4}$, then the number of zeros of Dirichlet $L$-functions attached to primitive characters modulo $q$ with $q \leq Q$ in the rectangle $R=\bigsqcup_{j=1}^{N} R_{j}$ is less than $C T(\log (Q T))^{-\alpha / 2}$. Therefore, if $T \geq T_{0}(\alpha)$ for a sufficiently large number $T_{0}(\alpha)$ depending only on $\alpha$, then the number of rectangles $R_{j}$ not having zeros of the Dirichlet $L$-functions is greater than $N-C T(\log (Q T))^{-\alpha / 2}(\geq N / 2)$.

Let $J$ be the set of all $j$ such that $R_{j}$ does not include zeros of those Dirichlet $L$-functions. From the above observation, we see that $\frac{N}{2} \leq \# J \leq$ 
$N$. By using the Euler product for $L(s, \chi)$ and the Taylor expansion for log, for $\sigma>1$, we find that

$$
\begin{aligned}
\log L(s, \chi) & =\sum_{p} \chi(p) p^{-s}+\frac{1}{2} \sum_{p} \chi(p)^{2} p^{-2 s}+\sum_{p} \sum_{n=3}^{\infty} \frac{\chi(p)^{n}}{n p^{n s}} \\
& =: P_{1}(s, \chi)+\frac{1}{2} P_{2}(s, \chi)+\Psi(s, \chi),
\end{aligned}
$$

and that $P_{2}(s, \chi)$ is regular on $\sigma>1 / 2$, and $\Psi(s, \chi)$ is regular on $\sigma>1 / 3$ and bounded on $\sigma \geq 1 / 2$. In addition, $\log L(s, \chi)$ is regular on $R_{j}(j \in J)$ since $L(s, \chi)$ does not have a zero on the same domain. Hence $P_{1}(s, \chi)$ is analytically continued to $R_{j}(j \in J)$.

Let $k$ be a positive integer. We define $a_{k, \chi}$ by

$$
\left(P_{1}(s, \chi)+\frac{1}{2} P_{2}(s, \chi)\right)^{k}=\sum_{n=1}^{\infty} \frac{a_{k, \chi}(n)}{n^{s}} .
$$

We can estimate $\left|a_{k, \chi}(n)\right|$ by the following way. If $a_{k, \chi}(n) \neq 0$, then $n$ can be written in the form $n=p_{1}^{l_{1}} \cdots p_{k}^{l_{k}}\left(l_{i} \in\{1,2\}\right)$. The number of ways which one can express $n$ by ordering $p_{1}^{l_{1}}, \ldots, p_{k}^{l_{k}}$ in different ways is at most $k$ !. This means that we have the inequality

$$
\left|a_{k, \chi}(n)\right| \leq k ! \leq k^{k}
$$

Hence, by the boundedness of $a_{k, \chi}(n)$ with respect to $n$,

$$
\sum_{n=1}^{\infty} \frac{a_{k, \chi}(n)}{n^{s}} \exp \left(-\frac{n}{X}\right)
$$

is an entire function for any $X>0$. Here, as our first step, we show that if $X=\left(Q^{2} T\right)^{1 / 4}$, and $k=[\alpha \log \log (Q T)]$, then we have

$$
\left(P_{1}(s, \chi)+\frac{1}{2} P_{2}(s, \chi)\right)^{k}=\sum_{n=1}^{\infty} \frac{a_{k, \chi}(n)}{n^{s}} \exp \left(-\frac{n}{X}\right)+O(1)
$$

for any $j \in J$ and $(\sigma, t) \in R_{j}^{\prime}$, where

$$
R_{j}^{\prime}=\left[\frac{1}{2}+14 k(\log \log (Q T))\left(\log \left(Q^{2} T\right)\right)^{-1}, 2\right] \times I_{j}^{\prime}
$$

with

$$
I_{j}^{\prime}=\left[T+2(j-1)(\log (Q T))^{D}+(\log (Q T))^{2}, T+2 j(\log (Q T))^{D}-(\log (Q T))^{2}\right] .
$$


The proof is as follows. Using a formula for Abelian weight (cf. [12, (5.25)]), we have

$$
\begin{aligned}
\sum_{n=1}^{\infty} \frac{a_{k, \chi}(n)}{n^{s}} & \exp \left(-\frac{n}{X}\right) \\
= & \frac{1}{2 \pi i} \int_{2-i \infty}^{2+i \infty}\left(P_{1}(s+w, \chi)+\frac{1}{2} P_{2}(s+w, \chi)\right)^{k} \Gamma(w) X^{w} \mathrm{~d} w .
\end{aligned}
$$

Moreover, we see that $\left|P_{1}(s+w, \chi)+\frac{1}{2} P_{2}(s+w, \chi)\right| \leq \log \zeta(2) \leq 1$, and that $|\Gamma(2+i u)| \ll|u|^{\frac{3}{2}} \exp \left(-\frac{\pi}{2}|u|\right)$ for $|u| \geq 1$ from the Stirling formula. These estimates yield that if $K=(\log (Q T))^{2}$,

$$
\begin{aligned}
\sum_{n=1}^{\infty} \frac{a_{k, \chi}(n)}{n^{s}} \exp \left(-\frac{n}{X}\right) & \\
=\frac{1}{2 \pi i} \int_{2-i K}^{2+i K}\left(P_{1}(s+w, \chi)\right. & \left.+\frac{1}{2} P_{2}(s+w, \chi)\right)^{k} \Gamma(w) X^{w} \mathrm{~d} w \\
& +O\left((\log (Q T))^{3} e^{-\pi(\log (Q T))^{2} / 2} X^{2}\right) .
\end{aligned}
$$

Here we consider the estimate of the integral on the right-hand side. By the Borel-Carathéodory lemma, we can find that the inequality

$$
|\log L(s, \chi)| \leq \frac{2|s-a|}{R-|s-a|} \max _{|s-a|=R} \operatorname{Re}(\log L(s, \chi))+\frac{R+|s-a|}{R-|s-a|}|\log L(a, \chi)|
$$

holds for $|s-a|<R$ with $R=2, a=\frac{5}{2}+k(\log \log (Q T))\left(\log \left(Q^{2} T\right)\right)^{-1}+i t, t \in$ $I_{j}{ }^{\prime}$. Hence we have

$$
|\log L(s, \chi)| \ll(\log (Q T))^{2}(\log \log (Q T))^{-2}
$$

for $\sigma \geq \frac{1}{2}+2 \alpha k(\log \log (Q T))\left(\log \left(Q^{2} T\right)\right)^{-1}, t \in I_{j}{ }^{\prime}$ since $L(s, \chi) \ll Q|t|$ holds for $\sigma \geq 1 / 2$.

By this estimate and the boundedness of $\Psi(s, \chi)$ for $\sigma \geq 1 / 2$, we have

$$
\begin{aligned}
\left|P_{1}(s, \chi)+\frac{1}{2} P_{2}(s, \chi)\right|^{k} & =|\log L(s, \chi)-\Psi(s, \chi)|^{k} \\
& \leq C^{k}(\log (Q T))^{2 k}(\log \log (Q T))^{-2 k}
\end{aligned}
$$


for $\sigma \geq \frac{1}{2}+2 \alpha k(\log \log (Q T))\left(\log \left(Q^{2} T\right)\right)^{-1}, t \in I_{j}{ }^{\prime}$. In addition, by the residue theorem, if $\beta=12 k(\log \log (Q T))\left(\log \left(Q^{2} T\right)\right)^{-1}$, then

$$
\begin{aligned}
\frac{1}{2 \pi i} \int_{2-i K}^{2+i K}\left(P_{1}(s+w, \chi)+\frac{1}{2} P_{2}(s+w, \chi)\right)^{k} \Gamma(w) X^{w} \mathrm{~d} w & \\
=\frac{1}{2 \pi i}\left(\int_{-\beta+i K}^{2+i K}+\int_{-\beta-i K}^{-\beta+i K}+\int_{2-i K}^{-\beta-i K}\right) & \\
& \left(P_{1}(s+w, \chi)+\frac{1}{2} P_{2}(s+w, \chi)\right)^{k} \Gamma(w) X^{w} \mathrm{~d} w \\
& +\left(P_{1}(s, \chi)+\frac{1}{2} P_{2}(s, \chi)\right)^{k}
\end{aligned}
$$

holds for $\sigma \geq \frac{1}{2}+14 k(\log \log (Q T))\left(\log \left(Q^{2} T\right)\right)^{-1}, t \in I_{j}^{\prime}$. By inequality (4.4) and the Stirling formula, we have

$$
\begin{aligned}
\frac{1}{2 \pi i} \int_{-\beta \pm i K}^{2 \pm i K}\left(P_{1}(s\right. & \left.+w, \chi)+\frac{1}{2} P_{2}(s+w, \chi)\right)^{k} \Gamma(w) X^{w} \mathrm{~d} w \\
& \ll C^{k}(\log (Q T))^{2 k+3}(\log \log (Q T))^{-2 k} e^{-\frac{\pi}{2}(\log (Q T))^{2}} X^{2},
\end{aligned}
$$

and

$$
\begin{aligned}
\frac{1}{2 \pi i} \int_{-\beta-i K}^{-\beta+i K}\left(P_{1}(s+w, \chi)\right. & \left.+\frac{1}{2} P_{2}(s+w, \chi)\right)^{k} \Gamma(w) X^{w} \mathrm{~d} w \\
& \ll \beta^{-1} X^{-\beta} C^{k}(\log (Q T))^{2 k}(\log \log (Q T))^{-2 k}
\end{aligned}
$$

From the above estimates, if $X=\left(Q^{2} T\right)^{1 / 4}$, and $k=[\alpha \log \log (Q T)]$, then we obtain the formula (4.3).

Next we consider the function

$$
F_{2 k}(T, \chi):=\sum_{j \in J} \max _{s \in R_{j}^{\prime}}|\log L(s, \chi)|^{2 k} .
$$

By (4.2) and (4.3), we have

$$
\begin{aligned}
|\log L(s, \chi)|^{2 k} & \leq 2^{2 k}\left(\left|P_{1}(s, \chi)+\frac{1}{2} P_{2}(s, \chi)\right|^{2 k}+|\Psi(s, \chi)|^{2 k}\right) \\
& =2^{2 k}\left|\sum_{n=1}^{\infty} \frac{a_{k, \chi}(n)}{n^{s}} \exp \left(-\frac{n}{X}\right)+O(1)\right|^{2}+|2 \Psi(s, \chi)|^{2 k} \\
& \leq 4^{2 k}\left|\sum_{n=1}^{\infty} \frac{a_{k, \chi}(n)}{n^{s}} \exp \left(-\frac{n}{X}\right)\right|^{2}+C^{k}
\end{aligned}
$$


Therefore, if $r=(\log \log (Q T))^{2}\left(\log \left(Q^{2} T\right)\right)^{-1}$, and $s_{j}$ is an element of $R_{j}^{\prime}$ satisfying

$$
\max _{s \in R_{j}^{\prime}}|\log L(s, \chi)|^{2 k}=\left|\log L\left(s_{j}, \chi\right)\right|^{2 k},
$$

then we have

$$
\begin{aligned}
\left|\log L\left(s_{j}, \chi\right)\right|^{2 k} & \ll 4^{2 k}\left|\sum_{n=1}^{\infty} \frac{a_{k, \chi}(n)}{n^{s_{j}}} \exp \left(-\frac{n}{X}\right)\right|^{2}+C^{2 k} \\
& =\frac{4^{2 k}}{\pi r^{2}}\left|\iint_{\left|s-s_{j}\right| \leq r}\left(\sum_{n=1}^{\infty} \frac{a_{k, \chi}(n)}{n^{s}} \exp \left(-\frac{n}{X}\right)\right)^{2} \mathrm{~d} \sigma \mathrm{d} t\right|+C^{2 k} \\
& \ll \frac{4^{2 k}}{r^{2}} \iint_{\left|s-s_{j}\right| \leq r}\left|\sum_{n=1}^{\infty} \frac{a_{k, \chi}(n)}{n^{s}} \exp \left(-\frac{n}{X}\right)\right|^{2} \mathrm{~d} \sigma \mathrm{d} t+C^{2 k}
\end{aligned}
$$

by the mean value theorem on analytic functions. By the disjointness of the domains $\left|s-s_{j}\right| \leq r$ for each $j$ and the estimate

$$
\begin{aligned}
& \left|\sum_{n>X^{2}} \frac{a_{k, \chi}(n)}{n^{s}} \exp \left(-\frac{n}{X}\right)\right| \leq k^{k} \sum_{n>X^{2}} \frac{1}{n^{1 / 2}} \exp \left(-\frac{n}{X}\right) \\
& \quad=k^{k} \sum_{m=0}^{\infty} \sum_{2^{m} X^{2}<n \leq 2^{m+1} X^{2}} \frac{1}{n^{1 / 2}} \exp \left(-\frac{n}{X}\right) \leq k^{k} \sum_{m=0}^{\infty} 2^{\frac{m}{2}} X \exp \left(-2^{m} X\right) \\
& \leq k^{k} X\left(\exp (-X)+\sum_{m=1}^{\infty}\left(2^{1 / 2} e^{-X}\right)^{m}\right) \ll k^{k} X \exp (-X),
\end{aligned}
$$

we have

$$
\begin{aligned}
F_{2 k}(T, \chi) \ll \frac{4^{2 k}}{r^{2}} \iint_{E} \mid \sum_{n \leq X^{2}} \frac{a_{k, \chi}(n)}{n^{s}} & \left.\exp \left(-\frac{n}{X}\right)\right|^{2} \mathrm{~d} \sigma \mathrm{d} t \\
& +\left(C^{2 k}+r^{-2}(4 k)^{2 k} X^{2} \exp (-2 X)\right) N,
\end{aligned}
$$

where $E$ is the domain with $\frac{1}{2} \leq \sigma \leq 2, T \leq t \leq 2 T$. We used the inequality $\# J \leq N$ in the above estimation. Recall that $N=\left[\frac{1}{2} T(\log (Q T))^{-D}\right]$. As 
for the remaining integral, we have

$$
\begin{aligned}
\mid \sum_{n \leq X^{2}} & \left.\frac{a_{k, \chi}(n)}{n^{s}} \exp \left(-\frac{n}{X}\right)\right|^{2}=\sum_{m, n \leq X^{2}} \frac{a_{k, \chi}(m) \overline{a_{k, \chi}(n)}}{m^{\sigma+i t} n^{\sigma-i t}} \exp \left(-\frac{m+n}{X}\right) \\
= & \left(\sum_{\substack{m, n \leq X^{2} \\
m \neq n}}+\sum_{\substack{m, n \leq X^{2} \\
m=n}} \frac{a_{k, \chi}(m) \overline{a_{k, \chi}(n)}}{m^{\sigma+i t} n^{\sigma-i t}} \exp \left(-\frac{m+n}{X}\right)\right. \\
= & \sum_{\substack{m, n \leq X^{2} \\
m \neq n}} \frac{a_{k, \chi}(m) \overline{a_{k, \chi}(n)}}{m^{\sigma+i t} n^{\sigma-i t}} \exp \left(-\frac{m+n}{X}\right)+O\left(k^{2 k} \sum_{n \leq X^{2}} \frac{1}{n^{2 \sigma}}\right),
\end{aligned}
$$

and

$$
\begin{aligned}
& \iint_{E}\left|\sum_{n \leq X^{2}} \frac{a_{k, \chi}(n)}{n^{s}} \exp \left(-\frac{n}{X}\right)\right|^{2} \mathrm{~d} s \\
& =\sum_{\substack{m, n \leq X^{2} \\
m \neq n}} a_{k, \chi}(m) \overline{a_{k, \chi}(n)} \exp \left(-\frac{m+n}{X}\right)\left(\int_{\frac{1}{2}}^{2} \frac{\mathrm{d} \sigma}{(m n)^{\sigma}}\right)\left(\int_{T}^{2 T}\left(\frac{n}{m}\right)^{i t} \mathrm{~d} t\right) \\
& \quad+O\left(k^{2 k} T \log X\right) \\
& \ll k^{2 k} \sum_{\substack{m, n \leq X^{2} \\
m \neq n}}\left|\log \left(\frac{n}{m}\right)\right|^{-1} \frac{1}{(m n)^{1 / 2}}+k^{2 k} T \log X \ll k^{2 k} X^{4}+k^{2 k} T \log X .
\end{aligned}
$$

Hence we find that

$$
\iint_{E}\left|\sum_{n \leq X^{2}} \frac{a_{k, \chi}(n)}{n^{s}} \exp \left(-\frac{n}{X}\right)\right|^{2} \mathrm{~d} s \ll k^{2 k} Q^{2} T \log X
$$

by $X=\left(Q^{2} T\right)^{1 / 4}$. Thus we have

$$
F_{2 k}(T, \chi) \ll r^{-2}(4 k)^{2 k} Q^{2} T \log X+\left(C^{2 k}+r^{-2}(4 k)^{2 k} X^{2} \exp (-2 X)\right) N,
$$

and there exists a $j_{0} \in J$ satisfying

$$
\begin{aligned}
\max _{s \in R_{j_{0}}^{\prime}}|\log L(s, \chi)|^{2 k} \ll r^{-2}(4 k)^{2 k} Q^{2}(\log X)(\log (Q T))^{\alpha / 3} & \\
& +C^{2 k}+r^{-2}(4 k)^{2 k} X^{2} \exp (-2 X)
\end{aligned}
$$

by the definition of $F$ and the inequality $\# J \geq \frac{N}{2}$. 
From the above discussion, the inequality

$$
\max _{s \in R_{j_{0}}^{\prime}}|\log L(s, \chi)| \ll k \ll \alpha \log \log (Q T)
$$

holds uniformly for $\chi \in S(Q)$, which completes the proof.

The following corollary is an immediate consequence of Proposition 3.

Corollary 1. We have

$$
\max _{\substack{\sigma \geq 1 / 2+14 \alpha r \\ t \in J_{0}, \chi \in S(Q)}}|L(s, \chi)|^{ \pm 1} \leq \exp (C \alpha \log \log (Q T))=(\log (Q T))^{C \alpha},
$$

where $C$ is a positive absolute constant, and the meaning of the other letters appearing in the formula is the same as in Proposition 3.

Lemma 2. Let $\chi$ be a primitive Dirichlet character modulo $q$. If $|t| \geq 1$ and $\sigma \leq \frac{1}{2}$, then

$$
|L(s, \chi)| \asymp\left(\frac{2 \pi e}{q|s|}\right)^{\sigma}(q|s|)^{1 / 2} \exp \left(|t| \tan ^{-1}\left(\frac{1-\sigma}{|t|}\right)\right)|L(1-s, \bar{\chi})| .
$$

If $|t| \leq 1, \sigma=-(m+1 / 2)$ with $m$ a positive integer, then

$$
|L(s, \chi)| \asymp\left(\frac{2 \pi e}{q|s|}\right)^{\sigma}(q|\sigma|)^{1 / 2}|L(1-s, \bar{\chi})| .
$$

Proof. By the functional equation for Dirichlet $L$-functions and the Stirling formula, we obtain this lemma.

Lemma 3. Let $J_{1}=\left[y_{1}, y_{2}\right]$ be the closed interval that is obtained by removing intervals of length $\log (Q T)$ from both ends of $J_{0}$. Then we have

$$
\max _{\substack{\sigma \geq 1 / 2-26 \alpha r \\ t \in J_{1}, \chi \in S(Q)}}|L(s, \chi)| \leq \exp \left(C \alpha(\log \log (Q T))^{2}\right),
$$

where $C$ is a positive absolute constant, and the meaning of the letters appearing in the formula is the same as in Proposition 3.

Proof. For $\sigma \geq 1 / 2+14 \alpha r, t \in J_{0}$, we have

$$
\begin{aligned}
|\log L(s, \bar{\chi})| & \geq \log |L(s, \bar{\chi})| \asymp \log \left|(q t)^{1 / 2-\sigma} L(1-s, \chi)\right| \\
& \geq \log |L(1-s, \chi)|-\left(\sigma-\frac{1}{2}\right) \log (q t)
\end{aligned}
$$

since $|L(s, \chi)| \asymp(q t)^{1 / 2-\sigma}|L(1-s, \bar{\chi})|$ by Lemma 2 . In addition, if $\chi$ is a primitive character, then $\bar{\chi}$ is also a primitive character. Therefore, for $t \in J_{0}$, we have

$$
\begin{aligned}
\log \left|L\left(\frac{1}{2}-26 \alpha r+i t, \chi\right)\right| & \ll \alpha r \log (Q T)+\left|\log \left(L\left(\frac{1}{2}+26 \alpha r+i t, \bar{\chi}\right)\right)\right| \\
& \ll \alpha(\log \log (Q T))^{2}
\end{aligned}
$$


by Proposition 3. Hence, if $t \in J_{0}$, then

$$
L\left(\frac{1}{2}-26 \alpha r+i t, \chi\right) \ll \exp \left(C \alpha(\log \log (Q T))^{2}\right) .
$$

Next we consider the function

$$
g_{l}(w)=L\left(s_{l}+w, \chi\right) e^{w^{2}} \quad(l=1,2),
$$

where $s_{l}=\frac{1}{2}+i y_{l}$. By a basic upper bound $L(s, \chi) \ll q(|t|+1)$ for $\sigma \geq 1 / 4$, we have

$$
\begin{aligned}
g_{l}(x \pm i \log (Q T)) & \ll e^{x^{2}-(\log (Q T))^{2}}\left|L\left(s_{l}+x \pm i \log (Q T), \chi\right)\right| \\
& \ll e^{x^{2}-(\log (Q T))^{2}} Q T \ll 1
\end{aligned}
$$

for $-26 \alpha r \leq x \leq 26 \alpha r, T \geq T_{0}(\alpha)$. Moreover, by estimates (4.5) and (4.8), we also have

$$
\begin{aligned}
g_{l}( \pm 26 \alpha r+i y) & =L\left(s_{l} \pm 26 \alpha r+i y, \chi\right) e^{\left(\frac{1}{2} \pm 26 \alpha r+i y\right)^{2}} \\
& \ll \exp \left(C \alpha(\log \log (Q T))^{2}\right)
\end{aligned}
$$

for $-\log (Q T) \leq y \leq \log (Q T)$. Hence, by the maximum modulus principle, we obtain

$$
g_{l}(x+i y) \ll \exp \left(C \alpha(\log \log (Q T))^{2}\right)
$$

for $-26 \alpha r \leq x \leq 26 \alpha r$ and $-\log (Q T) \leq y \leq \log (Q T)$. In particular, if $y=0$, then

$$
\begin{aligned}
L\left(s_{l}+x, \chi\right)=g_{l}(x) e^{x^{2}} & \ll \exp \left(C \alpha(\log \log (Q T))^{2}+x^{2}\right) \\
& \ll \exp \left(C \alpha(\log \log (Q T))^{2}\right)
\end{aligned}
$$

holds for $-26 \alpha r \leq x \leq 26 \alpha r$. Again by using the maximum modulus principle, we can find that

$$
L(s, \chi) \ll \exp \left(C \alpha(\log \log (Q T))^{2}\right)
$$

in the compact set $\frac{1}{2}-26 \alpha r \leq \sigma \leq \frac{1}{2}+26 \alpha r, t \in J_{1}$.

Lemma 4. If $f$ is a regular function and

$$
\left|\frac{f(s)}{f\left(s_{0}\right)}\right|<e^{M}, \quad(M>1)
$$

in $\left|s-s_{0}\right| \leq r$, then for any constant $0<\varepsilon<\frac{1}{2}$,

$$
\frac{f^{\prime}}{f}(s)=\sum_{\left|\rho-s_{0}\right| \leq \frac{1}{2} r} \frac{1}{s-\rho}+O_{\varepsilon}\left(\frac{M}{r}\right)
$$

in $\left|s-s_{0}\right| \leq\left(\frac{1}{2}-\varepsilon\right) r$, where $\rho$ is a zero of $f$.

Proof. This is Lemma 3 in [16]. 
Proposition 4. Let $J_{2}$ be the closed interval that is obtained by removing intervals of length $40 \alpha r$ from both ends of $J_{1}$. If $s_{0}=\frac{1}{2}+14 \alpha r+i t_{0}, t_{0} \in J_{2}$, then we have

$$
\frac{L^{\prime}(s, \chi)}{L(s, \chi)}=\sum_{\left|\rho-s_{0}\right| \leq 20 \alpha r} \frac{1}{s-\rho}+O(\log (Q T))
$$

for $\left|s-s_{0}\right| \leq 15 \alpha r$, where the meaning of the letters appearing in the following formula is the same as the above situations.

Proof. By Corollary 1, we have

$$
|L(s, \chi)| \geq \exp (-C \alpha \log \log (Q T))
$$

for $\sigma \geq \frac{1}{2}+14 \alpha r, t \in J_{0}$. By this inequality and Lemma 3, we find that

$$
\left|\frac{L(s, \chi)}{L\left(s_{0}, \chi\right)}\right| \leq \exp \left(C \alpha(\log \log (Q T))^{2}\right)
$$

for $\left|s-s_{0}\right| \leq 40 \alpha r$. Hence, by Lemma 4 with $\varepsilon=\frac{1}{8}$, we obtain

$$
\begin{aligned}
\frac{L^{\prime}(s, \chi)}{L(s, \chi)} & =\sum_{\left|\rho-s_{0}\right| \leq 20 \alpha r} \frac{1}{s-\rho}+O\left((40 \alpha r)^{-1} C \alpha(\log \log (Q T))^{2}\right) \\
& =\sum_{\left|\rho-s_{0}\right| \leq 20 \alpha r} \frac{1}{s-\rho}+O(\log (Q T))
\end{aligned}
$$

for $\left|s-s_{0}\right| \leq 15 \alpha r$.

Lemma 5. Let $t_{0} \in J_{2}$. If $s_{0}=\frac{1}{2}+14 \alpha r+i t_{0}$, then

$$
\begin{aligned}
\log L\left(\sigma+i t_{0}, \chi\right)=\sum_{\left|\rho-s_{0}\right| \leq 20 \alpha r}\left(\log \left(\sigma+i t_{0}-\rho\right)\right. & \left.-\log \left(s_{0}-\rho\right)\right) \\
& +O\left(\alpha(\log \log (Q T))^{2}\right)
\end{aligned}
$$

for $\frac{1}{2}-\alpha r \leq \sigma \leq \frac{1}{2}+29 \alpha r$. In particular, by taking the real parts on the both sides, we find that

(4.10) $\log \left|L\left(\sigma+i t_{0}, \chi\right)\right|$

$$
=\sum_{\left|\rho-\sigma_{0}\right| \leq 20 \alpha r} \log \left|\frac{\sigma+i t_{0}-\rho}{s_{0}-\rho}\right|+O\left(\alpha(\log \log (Q T))^{2}\right) .
$$


Proof. Let $t_{0} \in J_{2}$. Then by formula (4.9) for $\frac{1}{2} \leq \sigma \leq \frac{1}{2}+\frac{3}{2} \alpha r$, we find that

$$
\begin{aligned}
& \int_{\frac{1}{2}+14 \alpha r}^{\sigma} \frac{L^{\prime}\left(x+i t_{0}, \chi\right)}{L\left(x+i t_{0}, \chi\right)} \mathrm{d} x \\
& \quad=\sum_{\left|\rho-s_{0}\right| \leq 20 \alpha r} \int_{\frac{1}{2}+14 \alpha r}^{\sigma} \frac{\mathrm{d} x}{x+i t_{0}-\rho}+O\left(\alpha(\log \log (Q T))^{2}\right),
\end{aligned}
$$

and that

$$
\begin{aligned}
& \log L\left(\sigma+i t_{0}, \chi\right)-\log L\left(s_{0}, \chi\right) \\
& =\sum_{\left|\rho-s_{0}\right| \leq 20 \alpha r}\left(\log \left(\sigma+i t_{0}-\rho\right)-\log \left(s_{0}-\rho\right)\right)+O\left(\alpha(\log \log (Q T))^{2}\right) .
\end{aligned}
$$

Hence, by Proposition 3, we obtain

$$
\begin{aligned}
& \log L\left(\sigma+i t_{0}, \chi\right)=\sum_{\left|\rho-s_{0}\right| \leq 20 \alpha r}\left(\log \left(\sigma+i t_{0}-\rho\right)-\log \left(s_{0}-\rho\right)\right) \\
& +O\left(\alpha(\log \log (Q T))^{2}\right) \text {. }
\end{aligned}
$$

This completes the proof of Lemma 5 .

Now, let us start the proof of Proposition 1.

Proof of Proposition 1. If $\frac{1}{2}+14 \alpha r \leq \sigma \leq 2$, then Proposition 1 is implied by Proposition 3. Hence we consider the case $\frac{1}{2} \leq \sigma \leq \frac{1}{2}+14 \alpha r$. We find that

$$
\sum_{\left|\rho-s_{0}\right| \leq 20 \alpha r} \log \left|\frac{\sigma+i t_{0}-\rho}{s_{0}-\rho}\right| \geq \sum_{\left|\rho-s_{0}\right| \leq 20 \alpha r} \log \left|\frac{t_{0}-\gamma}{20 \alpha r}\right| \geq \sum_{\left|t_{0}-\gamma\right| \leq 20 \alpha r} \log \left|\frac{t_{0}-\gamma}{20 \alpha r}\right|
$$

hold uniformly for $\frac{1}{2} \leq \sigma \leq \frac{1}{2}+14 \alpha r$. Here, the above sums runs over nontrivial zeros $\rho$ of $L(s, \chi)$. In addition, if $\left[t_{1}, t_{1}+1\right] \subset J_{1}$, then for $T \geq$ $T_{0}(\alpha)$, we see that

$$
\begin{aligned}
& \int_{t_{1}}^{t_{1}+1} \sum_{|t-\gamma| \leq 20 \alpha r} \log \left|\frac{t-\gamma}{20 \alpha r}\right| \mathrm{d} t \\
& \quad=\sum_{t_{1}-20 \alpha r \leq \gamma \leq t_{1}+1+20 \alpha r} \int_{\max \left\{t_{1}, \gamma-20 \alpha r\right\}}^{\min \left\{t_{1}+1, \gamma+20 \alpha r\right\}} \log \left|\frac{t-\gamma}{20 \alpha r}\right| \mathrm{d} t \\
& \geq \sum_{t_{1}-20 \alpha r \leq \gamma \leq t_{1}+1+20 \alpha r} \int_{\gamma-20 \alpha r}^{\gamma+20 \alpha r} \log \left|\frac{t-\gamma}{20 \alpha r}\right| \mathrm{d} t \\
&=20 \alpha r \sum_{t_{1}-20 \alpha r \leq \gamma \leq t_{1}+1+20 \alpha r} \int_{-1}^{1} \log |x| \mathrm{d} x \\
& \geq-C \alpha r \log (Q T) \geq-C \alpha(\log \log (Q T))^{2},
\end{aligned}
$$


uniformly for $\chi \in S(Q)$. Remark that the above second inequality is obtained by the negativity of the integrand, and the last inequality is obtained by the estimate of the number of nontrivial zeros $\sum_{|t-\gamma| \leq 1+20 \alpha r} 1 \ll$ $\log (Q t)$, which is a consequence the Riemann-von Mangoldt formula for $L(s, \chi)$ (see [12, Corollary 14.7]). Hence, there exists a $t_{2} \in\left[t_{1}, t_{1}+1\right]$ satisfying

$$
\sum_{\left|\rho-s_{0}\right| \leq 20 \alpha r} \log \left|\frac{\sigma+i t_{2}-\rho}{\frac{1}{2}+\alpha r+i t_{2}-\rho}\right|^{-1} \ll \alpha(\log \log (Q T))^{2},
$$

uniformly for $\frac{1}{2} \leq \sigma \leq 2$ and $\chi \in S(Q)$. Thus this estimate implies Proposition 1 by formula (4.10).

\section{The finite Euler product appearing in the expression of Dirichlet $\boldsymbol{L}$-functions attached to imprimitive characters}

In this section, we are going to show some estimates on the function

$$
F_{q, \chi^{*}}(s)=\prod_{p \mid q}\left(1-\frac{\chi^{*}(p)}{p^{s}}\right)
$$

including Proposition 2. In the following, we consider the case $F_{q, \chi^{*}} \not \equiv 1$. In other words, we assume that $\chi^{*}$ is a primitive character modulo $d$ such that there exists a prime factor $p$ of $q$ with $p \nmid d$.

Lemma 6. Let $q \geq 2$. Then $F_{q, \chi^{*}}$ is an entire function of order 1 .

Proof. By the definition of $F_{q, \chi^{*}}$, if $\sigma<0$, then we have

$$
\begin{aligned}
\left|F_{q, \chi^{*}}(s)\right| & =\prod_{p \mid q}\left|1-\frac{\chi^{*}(p)}{p^{s}}\right| \leq \prod_{p \mid q}\left(1+\frac{1}{p^{\sigma}}\right)=\exp \left(\sum_{p \mid q} \log \left(1+\frac{1}{p^{\sigma}}\right)\right) \\
& \leq \exp \left(-2 \sigma \sum_{p \mid q} \log p\right) \leq \exp (2(\log q)|s|) .
\end{aligned}
$$

On the other hand, if $\sigma \geq 0$, then we have

$$
\left|F_{q, \chi^{*}}(s)\right|=\prod_{p \mid q}\left|1-\frac{\chi^{*}(p)}{p^{s}}\right| \leq 2^{\omega(q)} \ll_{q} 1 .
$$

Therefore, the order of $F_{q, \chi^{*}}$ is less than or equal to 1 . In addition, order of $F_{q, \chi^{*}}$ is greater than or equal to 1 since we can get the following lower 
bound

$$
\begin{aligned}
\left|F_{q, \chi^{*}}(s)\right| & =\prod_{p \mid q}\left|1-\frac{\chi^{*}(p)}{p^{s}}\right| \geq \prod_{\substack{p \mid q \\
p \nmid d}}\left(\frac{1}{p^{\sigma}}-1\right)=\exp \left(\sum_{p \mid q, p \nmid d} \log \left(\frac{1}{p^{\sigma}}-1\right)\right) \\
& \geq \exp \left(-\frac{1}{2} \sigma \sum_{p \mid q, p \nmid d} \log p\right) \geq \exp \left(\frac{1}{2}(\log 2)|\sigma|\right)
\end{aligned}
$$

for $\sigma \leq-2$. Hence we obtain Lemma 6 .

The next lemma is an immediate consequence of this lemma.

Lemma 7. Let $q \geq 2$ be an integer, $d$ be a proper divisor of $q$, and $\chi^{*}$ be a primitive Dirichlet character modulo d. Then we have

$$
F_{q, \chi^{*}}(s)=s^{r} e^{a+b s} \prod_{\eta}\left(1-\frac{s}{i \eta}\right) e^{s / i \eta},
$$

where the above infinite product runs over all the zeros of $F_{q, \chi^{*}}$ removing zero at $s=0$, and $r$ is the multiplicity of zero of $F_{q, \chi^{*}}$ at $s=0$. In particular, $r$ equals the number of prime factors of $q$ satisfying $\chi^{*}(p)=1$. Moreover, by taking the logarithmic derivative of both sides, we find that

$$
\frac{F_{q, \chi^{*}}^{\prime}}{F_{q, \chi^{*}}}(s)=\frac{r}{s}+b+\sum_{\eta}\left(\frac{1}{s-i \eta}+\frac{1}{i \eta}\right) .
$$

Lemma 8. If $b$ is the number appearing in (5.1), then

$$
b=-\frac{1}{2} \log \left(q^{\prime} / d^{\prime}\right)+i \sum_{\substack{p \mid q, p \nmid d \\ \chi^{*}(p) \neq 1}} \frac{\operatorname{Im}\left(\chi^{*}(p)\right)}{2-2 \operatorname{Re}\left(\chi^{*}(p)\right)} \log p
$$

where $q^{\prime}=\operatorname{rad}(q), d^{\prime}=\operatorname{rad}(d)$.

Proof. By formula (5.2), $b$ can be expressed by

$$
\lim _{\sigma \downarrow 0}\left(\frac{F_{q, \chi^{*}}^{\prime}}{F_{q, \chi^{*}}}(\sigma)-\frac{r}{\sigma}\right)=b .
$$

On the other hand, by taking logarithmic derivatives in (3.2), we have

$$
\frac{F_{q, \chi^{*}}^{\prime}}{F_{q, \chi^{*}}}(s)=\sum_{p \mid q} \frac{\chi^{*}(p) \log p}{p^{s}-\chi^{*}(p)}=\sum_{i=1}^{r} \frac{\log p_{i}}{p_{i}^{s}-1}+\sum_{\substack{p \mid q \\ \chi^{*}(p) \neq 1}} \frac{\chi^{*}(p) \log p}{p^{s}-\chi^{*}(p)},
$$

where $p_{i}$ are prime factors of $q$ with $\chi^{*}(p)=1$. By the Taylor expansion of exp, we also see that

$$
\lim _{\sigma \downarrow 0}\left(\frac{\log p_{i}}{p_{i}^{\sigma}-1}-\frac{1}{\sigma}\right)=\lim _{\sigma \downarrow 0}\left(\frac{1}{\sigma+\frac{1}{2} \sigma^{2} \log p_{i}+O\left(\sigma^{3}\right)}-\frac{1}{\sigma}\right)=-\frac{1}{2} \log p_{i} .
$$


Therefore, we obtain

$$
\lim _{\sigma \downarrow 0}\left(\frac{F_{q, \chi^{*}}^{\prime}}{F_{q, \chi^{*}}}(\sigma)-\frac{r}{\sigma}\right)=-\frac{1}{2} \sum_{i=1}^{r} \log p_{i}+\sum_{\substack{p \mid q \\ \chi^{*}(p) \neq 1}} \frac{\chi^{*}(p) \log p}{1-\chi^{*}(p)} .
$$

Moreover, if $\chi^{*}(p) \neq 0,1$, then we can find that the identity

$$
\frac{\chi^{*}(p)}{1-\chi^{*}(p)}=-\frac{1}{2}+i \frac{\operatorname{Im}\left(\chi^{*}(p)\right)}{2-2 \operatorname{Re}\left(\chi^{*}(p)\right)}
$$

holds by easy calculations. Thus we obtain Lemma 8 .

Lemma 9. Let $h>0$, and $N_{q, \chi^{*}}(t, h)$ be the number of zeros in of $F_{q, \chi^{*}}$ with $t \leq \eta \leq t+h$. For any $t \in \mathbb{R}$, we have

$$
N_{q, \chi^{*}}(t, h) \leq 2 \omega\left(q^{\prime} / d^{\prime}\right)+h \log \left(q^{\prime} / d^{\prime}\right)+\frac{2 h^{2}}{h^{2}+t^{2}} r,
$$

where $q^{\prime}=\operatorname{rad}(q)$ and $d^{\prime}=\operatorname{rad}(d)$.

Proof. By formula (5.2), we have

$$
\frac{F_{q, \chi^{*}}^{\prime}}{F_{q, \chi^{*}}}(h+i t)=\frac{r}{h+i t}+b+\sum_{\eta}\left(\frac{1}{h+i t-i \eta}+\frac{1}{i \eta}\right) .
$$

On the other hand, by taking logarithmic derivatives in (3.2), we find that

$$
\begin{aligned}
\left|\frac{F_{q, \chi^{*}}^{\prime}}{F_{q, \chi^{*}}}(h+i t)\right|=\left|\sum_{p \mid q} \frac{\chi^{*}(p) \log p}{p^{h+i t}-\chi^{*}(p)}\right| \leq \sum_{\substack{p \mid q \\
p \nmid d}} \frac{\log p}{p^{h}-1} \leq \sum_{\substack{p \mid q \\
p \nmid d}} \frac{1}{h} \\
=h^{-1} \omega\left(q^{\prime} / d^{\prime}\right) .
\end{aligned}
$$

Now, we take the real parts of the both sides of (5.4). Then, by $\operatorname{Re} \sum_{\eta}(i \eta)^{-1}=0$ and (5.3), we have

$$
\sum_{\eta} \frac{h}{h^{2}+(t-\eta)^{2}} \leq \frac{\omega\left(q^{\prime} / d^{\prime}\right)}{h}+\frac{1}{2} \log \left(q^{\prime} / d^{\prime}\right)+\frac{h}{h^{2}+t^{2}} r .
$$

Hence, we have

$$
\begin{aligned}
\frac{\omega\left(q^{\prime} / d^{\prime}\right)}{h}+\frac{1}{2} \log \left(q^{\prime} / d^{\prime}\right)+\frac{h}{h^{2}+t^{2}} r & \geq \sum_{\eta} \frac{h}{h^{2}+(t-\eta)^{2}} \\
\geq \sum_{|t-\eta+h / 2| \leq h / 2} \frac{h}{h^{2}+(t-\eta)^{2}} & \geq \sum_{|t-\eta+h / 2| \leq h / 2} \frac{1}{2 h}=\frac{1}{2 h} N_{q, \chi^{*}}(t, h),
\end{aligned}
$$

which completes the proof of Lemma 9. 
Proposition 5. Let $h>0$. If $|\sigma| \leq h$, then we have

$$
\frac{F_{q, \chi^{*}}^{\prime}}{F_{q, \chi^{*}}}(s)=\frac{r}{s}+\sum_{|t-\eta| \leq h} \frac{1}{s-i \eta}+O\left(h^{-1} \omega\left(q^{\prime} / d^{\prime}\right)+\log \left(q^{\prime} / d^{\prime}\right)+\frac{r}{|t|+h}\right) .
$$

Proof. By (5.2) and (5.5), we find that

$$
\begin{aligned}
& \frac{F_{q, \chi^{*}}^{\prime}}{F_{q, \chi^{*}}}(\sigma+i t)-\frac{F_{q, \chi^{*}}^{\prime}}{F_{q, \chi^{*}}}(h+i t) \\
& \quad=\frac{r}{\sigma+i t}-\frac{r}{h+i t}+\sum_{\eta}\left(\frac{1}{\sigma+i t-i \eta}-\frac{1}{h+i t-i \eta}\right),
\end{aligned}
$$

and that $\left|\frac{F_{q, \chi^{*}}^{\prime}}{F_{q, \chi^{*}}}(h+i t)\right| \leq h^{-1} \omega\left(q^{\prime} / d^{\prime}\right)$. Therefore, we have

$$
\begin{array}{r}
\frac{F_{q, \chi^{*}}^{\prime}}{F_{q, \chi^{*}}}(\sigma+i t)=\frac{r}{\sigma+i t}+\left(\sum_{|t-\eta| \leq h}+\sum_{|t-\eta|>h}\right) \\
+O\left(\frac{1}{\sigma+i t-i \eta}-\frac{1}{h+i t-i \eta}\right) \\
+O\left(\frac{r}{|t|+h}+h^{-1} \omega\left(q^{\prime} / d^{\prime}\right)\right) .
\end{array}
$$

In addition, we can obtain that

$$
\begin{aligned}
& \sum_{|t-\eta|>h}\left(\frac{1}{\sigma+i t-i \eta}-\frac{1}{h+i t-i \eta}\right) \\
&= \sum_{|t-\eta|>h} \frac{h-\sigma}{(\sigma+i t-i \eta)(h+i t-i \eta)} \ll \sum_{|t-\eta|>h} \frac{h}{|t-\eta|^{2}} \ll \sum_{\eta} \frac{h}{h^{2}+(t-\eta)^{2}} \\
& \leq h^{-1} \omega\left(q^{\prime} / d^{\prime}\right)+\frac{1}{2} \log \left(q^{\prime} / d^{\prime}\right)+\frac{h}{h^{2}+t^{2}} r
\end{aligned}
$$

by (5.6), and that

$$
\sum_{|t-\eta| \leq h} \frac{1}{h+i t-i \eta} \ll \sum_{|t-\eta| \leq h} h^{-1} \ll h^{-1} \omega\left(q^{\prime} / d^{\prime}\right)+\log \left(q^{\prime} / d^{\prime}\right)+\frac{h}{h^{2}+t^{2}} r
$$

by Lemma 9 . Hence we obtain Proposition 5 .

Proposition 6. Let $h>0$. For $|\sigma| \leq h,|t| \geq h$, we have

(5.7) $\log F_{q, \chi^{*}}(\sigma+i t)=\frac{1}{2} \sum_{|t-\eta| \leq h} \log \left(\frac{\sigma^{2}+(t-\eta)^{2}}{h^{2}+(t-\eta)^{2}}\right)$

$$
+O\left(h^{-1} \omega\left(q^{\prime} / d^{\prime}\right)+h \log \left(q^{\prime} / d^{\prime}\right)+\frac{h}{|t|} r\right) \text {. }
$$


Proof. By Lemma 9 and Proposition 5, we can find that

$$
\begin{aligned}
\log F_{q, \chi^{*}}(\sigma+i t)= & \int_{h}^{\sigma} \frac{F_{q, \chi^{*}}^{\prime}}{F_{q, \chi^{*}}}(\alpha+i t) \mathrm{d} \alpha+\log F_{q, \chi^{*}}(h+i t) \\
= & \frac{1}{2} \sum_{|t-\eta| \leq h} \log \left(\frac{\sigma^{2}+(t-\eta)^{2}}{h^{2}+(t-\eta)^{2}}\right)+\log F_{q, \chi^{*}}(h+i t) \\
& \quad+O\left(\omega\left(q^{\prime} / d^{\prime}\right)+h \log \left(q^{\prime} / d^{\prime}\right)+\frac{h}{|t|} r\right)
\end{aligned}
$$

In addition, we see that

$$
\begin{aligned}
\left|\log F_{q, \chi^{*}}(h+i t)\right| & =\left|\sum_{\substack{p \mid q \\
p \nmid d}} \sum_{n=1}^{\infty} \frac{1}{n}\left(\frac{\chi^{*}(p)}{p^{h+i t}}\right)^{n}\right| \leq \sum_{\substack{p \mid q \\
p \nmid d}} \sum_{n=1}^{\infty} \frac{1}{p^{h n}}=\sum_{\substack{p \mid q \\
p \nmid d}} \frac{1}{p^{h}-1} \\
& \leq \sum_{\substack{p \mid q \\
p \nmid d}} \frac{1}{h \log p} \ll h^{-1} \omega\left(q^{\prime} / d^{\prime}\right)
\end{aligned}
$$

by the definition of $F_{q, \chi^{*}}$ and the Taylor expansion of the logarithmic function. Hence we obtain Proposition 6 .

Now, let us start the proof of Proposition 2.

Proof of Proposition 2. Let $T \geq \omega(q)$, and $d$ be the smallest modulus of $\chi \in S_{1}(q)$. Then, by Lemma 9 , the number of zeros $i \eta$ of the all $F_{q, \chi^{*}}$ with $\chi \in S_{1}(q)$ with $\eta \in[T, T+1]$ is less than $C \# S_{1}(q) \log \left(q^{\prime} / d^{\prime}\right)$, where $C$ is an absolute positive constant. Therefore, there exists a $t_{0} \in[T, T+1]$ such that $\left|t_{0}-\eta\right| \geq \frac{1}{2 C \# S_{1}(q) \log \left(q^{\prime} / d^{\prime}\right)}$ holds for all zeros $i \eta$. Now we apply Proposition 6 with $h \asymp \sqrt{\frac{\omega\left(q^{\prime} / d^{\prime}\right) / \log \left(q^{\prime} / d^{\prime}\right)}{\log \left(\# S_{1}(q) \omega\left(q^{\prime} / d^{\prime}\right)+2\right)}}$. By taking real parts on the both sides of equation (5.7), we obtain

$$
\begin{gathered}
\log \left|F_{q, \chi^{*}}\left(\sigma+i t_{0}\right)\right| \geq-\frac{1}{2} \sum_{\left|t_{0}-\eta\right| \leq h} \log \left(8 C^{2} h^{2} \# S_{1}(q)^{2}\left(\log \left(q^{\prime} / d^{\prime}\right)\right)^{2}\right) \\
\geq-C^{\prime}\left(h^{-1} \omega\left(q^{\prime} / d^{\prime}\right)+h \log \left(q^{\prime} / d^{\prime} / d^{\prime}\right)\right) \\
\quad \times\left(1+\sqrt{\frac{\log \left(\# S_{1}(q) \omega\left(q^{\prime} / d^{\prime}\right)+2\right)}{\log \left(q^{\prime} / d^{\prime}\right) / \omega\left(q^{\prime} / d^{\prime}\right)}}\right),
\end{gathered}
$$


uniformly for $\chi \in S_{1}(q)$ and $|\sigma| \leq h$ with $C^{\prime}, C^{\prime \prime}>0$ sufficiently large positive absolute constants. Hence, we have

$$
\begin{array}{r}
\left|F_{q, \chi^{*}}\left(\sigma+i t_{0}\right)\right|^{-1} \leq \exp \left(C^{\prime \prime} \omega\left(q^{\prime} / d^{\prime}\right) \log \left(\# S_{1}(q) \omega\left(q^{\prime} / d^{\prime}\right)+2\right)\right. \\
\left.\times\left(1+\sqrt{\frac{\log \left(q^{\prime} / d^{\prime}\right) / \omega\left(q^{\prime} / d^{\prime}\right)}{\log \left(\# S_{1}(q) \omega\left(q^{\prime} / d^{\prime}\right)+2\right)}}\right)\right),
\end{array}
$$

which completes the proof of Proposition 2 .

\section{Proofs of Theorems 2, 4, and 5}

It suffices to prove Theorems 4,5 since Theorem 2 is an immediate consequence of these by equation (1.3).

Proof of Theorem 4. Let $x>0, T \geq \max \left\{T_{0}, \exp \left(q^{1 / 3}\right), 2 / x\right\}$, and $\sigma_{0}=$ $1+1 / \log (x+3)$. First, using Perron's formula (cf. [12, Theorem 5.2 and Corollary 5.3]), we have

$$
\begin{aligned}
& M^{*}(x, \chi)=\frac{1}{2 \pi i} \int_{\sigma_{0}-i T_{\nu}}^{\sigma_{0}+i T_{\nu}} \frac{x^{s}}{L(s, \chi) s} \mathrm{~d} s \\
& \quad+O\left(\frac{x \log (x+3)}{T}+\min \left\{1, \frac{x}{T\langle x\rangle}\right\}\right),
\end{aligned}
$$

where $T_{\nu}$ satisfies the inequality

$$
\left|L\left(\sigma+i T_{\nu}, \chi\right)\right|^{-1} \leq \exp \left(C(\log \log T)^{2}\right)
$$

for any $\frac{1}{2} \leq \sigma \leq 2$ and $\chi \in S(q)$ with $T_{\nu} \in[T, 2 T]$. Note that we can take the above $T_{\nu}$ by Proposition 1 . Here, we remark that $T \geq \exp \left(q^{1 / 3}\right) \gg q$.

Let $M=m+\frac{1}{2}$ with a positive integer $m$ satisfying $m>T$. By the residue theorem, we have

$$
\begin{aligned}
\frac{1}{2 \pi i} \int_{\sigma_{0}-i T_{\nu}}^{\sigma_{0}+i T_{\nu}} \frac{x^{s}}{L(s, \chi) s} \mathrm{~d} s \\
=\frac{1}{2 \pi i}\left(\int_{-M+i T_{\nu}}^{\sigma_{0}+i T_{\nu}}+\int_{-M-i T_{\nu}}^{-M+i T_{\nu}}+\int_{\sigma_{0}-i T_{\nu}}^{-M-i T_{\nu}}\right) \frac{x^{s}}{L(s, \chi) s} \mathrm{~d} s \\
\quad+\sum_{|\gamma|<T_{*}} \operatorname{Res}_{s=\rho}\left(\frac{x^{s}}{L(s, \chi) s}\right)+\sum_{0 \leq l<M} \operatorname{Res}_{s=-l}\left(\frac{x^{s}}{L(s, \chi) s}\right) \\
=: J_{1}+J_{2}+J_{3}+\sum_{|\gamma|<T_{\nu}} \operatorname{Res}_{s=\rho}\left(\frac{x^{s}}{L(s, \chi) s}\right)+\sum_{0 \leq l<M} \operatorname{Res}_{s=-l}\left(\frac{x^{s}}{L(s, \chi) s}\right) .
\end{aligned}
$$


Here, by the basic formula for residues, we find that

$$
\operatorname{Res}_{s=\rho}\left(\frac{x^{s}}{L(s, \chi) s}\right)=\frac{1}{(m(\rho)-1) !} \lim _{s \rightarrow \rho} \frac{\mathrm{d}^{m(\rho)-1}}{\mathrm{~d} s^{m(\rho)-1}}\left((s-\rho)^{m(\rho)} \frac{x^{s}}{L(s, \chi) s}\right) .
$$

Now we estimate the integrals $J_{1}, J_{2}$ and $J_{3}$. By Lemma $2, J_{2}$ is evaluated by

$$
\begin{aligned}
\left|J_{2}\right|=\left|\int_{|t| \leq T_{\nu}} \frac{x^{-M+i t}}{L(-M+i t, \chi)(-M+i t)} \mathrm{d} t\right| \\
\quad \ll x^{-M} \int_{|t| \leq T_{\nu}}\left(\frac{2 \pi e}{M}\right)^{M} M^{-3 / 2} \mathrm{~d} t \ll\left(\frac{x}{2 \pi e}\right)^{-M} M^{-M-3 / 2} T .
\end{aligned}
$$

Therefore, we have

$$
\lim _{M \rightarrow \infty} J_{2}=0
$$

Next we estimate $J_{1}$. We put

$$
J_{1}=\frac{1}{2 \pi i}\left(\int_{1 / 2+i T_{\nu}}^{\sigma_{0}+i T_{\nu}}+\int_{-1+i T_{\nu}}^{1 / 2+i T_{\nu}}+\int_{-M+i T_{\nu}}^{-1+i T_{\nu}}\right) \frac{x^{s}}{L(s, \chi) s} \mathrm{~d} s=: J_{1}^{\prime}+J_{1}^{\prime \prime}+J_{1}^{\prime \prime \prime} .
$$

By Lemma 2 and estimate (6.2), we find that

$$
\begin{gathered}
\left|J_{1}^{\prime}\right| \ll \int_{1 / 2}^{\sigma_{0}} x T_{\nu}^{-1} \exp \left(C(\log \log T)^{2}\right) \mathrm{d} \sigma \ll \frac{x \exp \left(C(\log \log T)^{2}\right)}{T}, \\
\left|J_{1}{ }^{\prime \prime}\right| \ll \frac{\exp \left(C(\log \log T)^{2}\right)}{T^{3 / 2}} \int_{-1}^{1 / 2}\left(x T_{\nu}\right)^{\sigma} \mathrm{d} \sigma \ll \frac{x^{1 / 2} \exp \left(C(\log \log T)^{2}\right)}{T \log (x T)},
\end{gathered}
$$

and that

$$
\left|J_{1}^{\prime \prime \prime}\right| \ll \frac{1}{T^{3 / 2}} \int_{-M}^{-1}\left(x T_{\nu}\right)^{\sigma} \mathrm{d} \sigma \ll \frac{(x T)^{-1}}{T^{3 / 2} \log (x T)} .
$$

Hence we have

$$
J_{1} \ll \frac{x \exp \left(C(\log \log T)^{2}\right)}{T} .
$$

Similarly, we have

$$
J_{3} \ll \frac{x \exp \left(C(\log \log T)^{2}\right)}{T}
$$

since $L(\bar{s}, \chi)=\overline{L(s, \bar{\chi})}$ and $\bar{\chi}$ is also a primitive character. From the above estimates, we obtain Theorem 4 .

Proof of Theorem 5. First, we find that

$$
M^{*}(x, \chi)=\frac{1}{2 \pi i} \int_{\sigma_{0}-i T_{\nu}}^{\sigma_{0}+i T_{\nu}} \frac{x^{s}}{L(s, \chi) s} \mathrm{~d} s+O\left(\frac{x \log (x+3)}{T}+\min \left\{1, \frac{x}{T\langle x\rangle}\right\}\right),
$$


similarly to (6.1), where we use the same notation as in the proof of Theorem 4. Here, by the proof of Proposition $1, T_{\nu} \in J_{1} \subset J_{0}$ holds, where $J_{0}$ and $J_{1}$ are intervals appearing in Corollary 1 and Lemma 3 , respectively. We also consider a uniform estimate of $F_{q, \chi^{*}}(s)$ for $\chi \in S^{*}(q)$. Here $S^{*}(q)$ denotes the set of all imprimitive characters modulo $q$. If $h:=\frac{1}{\log q} \leq \sigma \leq 2$, then we have

$$
\begin{aligned}
& \prod_{p \mid q}\left|1-\frac{\chi^{*}(p)}{p^{s}}\right|^{-1} \leq \prod_{p \mid q}(\left.1+\frac{1}{p^{h}-1}\right) \leq \prod_{p \mid q}\left(1+\frac{1}{h \log p}\right) \\
& \leq \exp \left(\sum_{p \mid q} \frac{1}{h \log p}\right) \leq \exp (C \omega(q) \log q) .
\end{aligned}
$$

Therefore, by this estimate, (6.2) and Lemma 2, we obtain

$$
\begin{aligned}
& \left|L\left(\sigma+i T_{\nu}, \chi\right)\right|^{-1}=\left|L\left(\sigma+i T_{\nu}, \chi^{*}\right)\right|^{-1} \prod_{p \mid q}\left|1-\frac{\chi^{*}(p)}{p^{s}}\right|^{-1} \\
& \quad \leq \exp \left(C\left((\log \log T)^{2}+\omega(q) \log q\right)\right) \leq \exp \left(C(\log \log T)^{2}\right)
\end{aligned}
$$

for $h \leq \sigma \leq 2$.

Here, let $M=m+\frac{1}{2}$, with a positive integer $m$ satisfying $m>T$. By Proposition 2 and $\omega(q) \ll \frac{\log q}{\log \log (q+5)}$, we can also take some $T_{*} \in$ $\left[T_{\nu}, T_{\nu}+1\right] \subset J_{0}$ such that

$$
\left|F_{q, \chi^{*}}\left(\sigma+i T_{*}\right)\right|^{-1}=\left|\prod_{p \mid q}\left(1-\frac{\chi^{*}(p)}{p^{\sigma+i T_{*}}}\right)\right|^{-1} \leq \exp \left(\frac{C(\log q)^{2}}{\log \log (q+5)}\right)
$$

holds for $|\sigma| \leq h$, uniformly $\chi \in S^{*}(q)$. Then, by using the residue theorem, we have

$$
\begin{aligned}
& \frac{1}{2 \pi i} \int_{\sigma_{0}-i T_{\nu}}^{\sigma_{0}+i T_{\nu}} \frac{x^{s}}{L(s, \chi) s} \mathrm{~d} s \\
& =\frac{1}{2 \pi i} \times\left(\int_{1 / 4+i T_{\nu}}^{\sigma_{0}+i T_{\nu}}+\int_{1 / 4+i T_{*}}^{1 / 4+i T_{\nu}}+\int_{-M+i T_{*}}^{1 / 4+i T_{*}}+\int_{-M-i T_{*}}^{-M+i T_{*}}\right. \\
& \left.\quad+\int_{1 / 4-i T_{*}}^{-M-i T_{*}}+\int_{1 / 4-i T_{\nu}}^{1 / 4-i T_{*}}+\int_{\sigma_{0}+i T_{\nu}}^{1 / 4+i T_{\nu}}\right) \frac{x^{s}}{L(s, \chi) s} \mathrm{~d} s \\
& \quad+\sum_{|\gamma|<T_{\nu}} \operatorname{Res}_{s=\rho}\left(\frac{x^{s}}{L(s, \chi) s}\right)+\sum_{|\gamma|<T_{*}} \operatorname{Res}_{s=i \eta}\left(\frac{x^{s}}{L(s, \chi) s}\right)+\sum_{0 \leq l<M} \operatorname{Res}_{s=-l}\left(\frac{x^{s}}{L(s, \chi) s}\right) .
\end{aligned}
$$

Now, we can obtain the residues for non-positive integer in a similar manner as in Theorem 4 since the all trivial zeros of $L\left(s, \chi^{*}\right)$ are simple. 
We estimate the integrals. As for the first integral, by inequality (6.4), we find that

$$
\left|\int_{1 / 4+i T_{\nu}}^{\sigma_{0}+i T_{\nu}} \frac{x^{s}}{L(s, \chi) s} \mathrm{~d} s\right| \leq \frac{x}{T} \exp \left(C(\log \log T)^{2}\right) .
$$

Next we consider the fourth integral. Now, $F_{q, \chi^{*}}(s)$ is estimated by

$$
\left|F_{q, \chi^{*}}(\sigma+i t)\right|=\prod_{\substack{p \mid q \\ p \nmid b}}\left|1-\frac{\chi^{*}(p)}{p^{\sigma+i t}}\right| \geq \prod_{\substack{p \mid q \\ p \nmid b}}\left(p^{-\sigma}-1\right) \geq(|\sigma| \log 2)^{\omega(q)}
$$

for $\sigma \leq-h$. Here $b$ is the modulus of $\chi^{*}$. Therefore, by Lemma 2 and (6.6), the fourth integral is estimated by

$$
\begin{aligned}
\int_{|t| \leq T_{*}} \frac{x^{-M+i t}}{L(-M+i t, \chi)(-M+i t)} \mathrm{d} t & \ll x^{-M} \int_{|t| \leq T_{*}}\left(\frac{2 \pi e}{M}\right)^{M} M^{-3 / 2} \mathrm{~d} t \\
& \ll\left(\frac{x}{2 \pi e}\right)^{-M} M^{-M-3 / 2} T .
\end{aligned}
$$

The last term tends to zero as $M \rightarrow+\infty$. That is,

$$
\lim _{M \rightarrow \infty} \int_{|t| \leq T_{*}} \frac{x^{-M+i t}}{L(-M+i t, \chi)(-M+i t)} \mathrm{d} t=0 .
$$

Next we consider the second integral. Now, we can see $\left[T_{\nu}, T_{*}\right] \subset J_{0}$ since $T_{\nu} \in J_{1}$, and so we can apply Corollary 1 to the second integral. Hence, by Corollary 1, (4.6) and (6.3), we have

$$
\int_{1 / 4+i T_{*}}^{1 / 4+i T_{\nu}} \frac{x^{s}}{L(s, \chi) s} \mathrm{~d} s \ll \frac{x^{1 / 4}}{T}\left(q^{\omega(q)} \log T\right)^{C} \ll \frac{x^{1 / 4}}{T} \exp \left(C(\log \log T)^{2}\right) .
$$

Next we consider the third integral. We put

$$
\int_{-M+i T_{*}}^{1 / 4+i T_{*}} \frac{x^{s}}{L(s, \chi) s} \mathrm{~d} s=\left(\int_{-1+i T_{*}}^{1 / 4+i T_{*}}+\int_{-M+i T_{*}}^{-1+i T_{*}}\right) \frac{x^{s}}{L(s, \chi) s} \mathrm{~d} s .
$$

Here, we can also apply Corollary 1 to this case by $T_{*} \in J_{0}$. By Corollary 1 , (4.6), (6.3), (6.5) and (6.6), we can find that

$$
\begin{aligned}
\int_{-1+i T_{*}}^{1 / 4+i T_{*}} \frac{x^{s}}{L(s, \chi) s} \mathrm{~d} s & \ll \frac{\exp \left(C\left(\frac{(\log q)^{2}}{\log \log (q+5)}+\log \log T\right)\right)}{T^{3 / 2}} \int_{-1}^{1 / 4}(x T)^{\sigma} \mathrm{d} \sigma \\
& \ll \frac{x^{1 / 4} \exp \left(C(\log \log T)^{2}\right)}{T^{5 / 4} \log (x T)},
\end{aligned}
$$

and that

$$
\int_{-M+i T_{*}}^{-1+i T_{*}} \frac{x^{s}}{L(s, \chi) s} \mathrm{~d} s \ll \frac{1}{T^{3 / 2}} \int_{-M}^{-1}\left(x T_{*}\right)^{\sigma} \mathrm{d} \sigma \ll \frac{1}{x T^{5 / 2} \log (x T)} .
$$


From the above discussion, the first four integrals are estimated by

$$
\ll \frac{x \exp \left(C(\log \log T)^{2}\right)}{T} .
$$

The remaining integrals also have the same upper bound since $L(\bar{s}, \chi)=$ $\overline{L(s, \bar{\chi})}$ holds, and estimate (6.7) is uniform for $\chi \in S^{*}(q)$. Thus, we obtain estimate (2.2) for Theorem 5 .

\section{Estimates of residues and proof of Theorem 3}

Lemma 10. Let $x \geq 2$. Let $\chi$ be a primitive character modulo $d \geq 1$. Then, we have

$$
\operatorname{Res}_{s=0}\left(\frac{x^{s}}{L(s, \chi) s}\right) \ll \begin{cases}1 & \text { if } \chi(-1)=-1 \text { or } \chi \text { is principal, } \\ \log x & \text { otherwise. }\end{cases}
$$

Moreover, we have

$$
\sum_{l=1}^{\infty} \operatorname{Res}_{s=-l}\left(\frac{x^{s}}{L(s, \chi) s}\right) \ll \begin{cases}x^{-1} & \text { if } \chi(-1)=-1 \\ x^{-2} & \text { if } \chi(-1)=1 .\end{cases}
$$

The above implicit constants are absolute.

Proof. We have the formula by the functional equation

$$
L(s, \chi)=\varepsilon(\chi) L(1-s, \bar{\chi}) 2^{s} \pi^{s-1} d^{1 / 2-s} \Gamma(1-s) \sin \left(\frac{\pi}{2}(s+\kappa)\right),
$$

where $\kappa=1$ if $\chi(-1)=-1$ and $\kappa=0$ otherwise. Here $\varepsilon(\chi)$ is a number with $|\varepsilon(\chi)|=1$. When $d=1$, estimate (7.1) is clear. When $d \geq 2$, we obtain, using this functional equation, that

$$
\begin{aligned}
\operatorname{Res}_{s=0} & \left(\frac{x^{s}}{L(s, \chi) s}\right) \\
= & \begin{cases}\frac{\pi}{\varepsilon(\chi) L(1, \bar{\chi}) d^{1 / 2}} & \text { if } \chi(-1)=-1, \\
\frac{2}{\varepsilon(\chi) L(1, \bar{\chi}) d^{1 / 2}}\left(\log \left(\frac{d x}{2 \pi}\right)+\frac{L^{\prime}(1, \bar{\chi})}{L(1, \bar{\chi})}+\frac{\Gamma^{\prime}}{\Gamma}(1)\right) & \text { if } \chi(-1)=1 .\end{cases}
\end{aligned}
$$

Hence, by Siegel's theorem $L(1, \chi) \gg_{\varepsilon} q^{-\varepsilon}$ and the standard estimate $L^{\prime}(1, \chi) \ll(\log q)^{2}$, we have $(7.1)$.

We see that if $\chi(-1)=-1$,

$$
\begin{aligned}
& \operatorname{Res}_{s=-l}\left(\frac{x^{s}}{L(s, \chi) s}\right) \\
& \quad= \begin{cases}\frac{(-1)^{k} 2(d x / 2 \pi)^{-(2 k-1)}}{\varepsilon(\chi) d^{1 / 2} L(2 k, \bar{\chi})(2 k-1)(2 k-1) !} & \text { if } l \text { is odd with } l=2 k-1, \\
0 & \text { if } l \text { is even, }\end{cases}
\end{aligned}
$$


and that if $\chi(-1)=1$,

$\operatorname{Res}_{s=-l}\left(\frac{x^{s}}{L(s, \chi) s}\right)= \begin{cases}\frac{(-1)^{k}(d x / 2 \pi)^{-2 k}}{\varepsilon(\chi) d^{1 / 2} L(2 k+1, \bar{\chi}) k(2 k) !} & \text { if } l \text { is even with } l=2 k, \\ 0 & \text { if } l \text { is odd. }\end{cases}$

Hence, we also obtain (7.2)

Lemma 11. Let $q \geq 2, \chi$ be an imprimitive Dirichlet character modulo $q$, and $\chi^{*}$ be the primitive character modulo $b$ inducing $\chi$. Let $T \geq 3+\log q$. Put $q^{\prime}=\operatorname{rad}(q), b^{\prime}=\operatorname{rad}(b)$. Let $\kappa$ be the same number as in the proof of Lemma 10. If $x \geq q^{c} \exp \left(c(\log T)^{\frac{2}{3}}(\log \log T)^{\frac{1}{3}}\right)$ holds for a sufficiently large constant $c$, then we have

$$
\begin{aligned}
\sum_{|\eta|<T} \operatorname{Res}_{s=i \eta}\left(\frac{x^{s}}{L(s, \chi) s}\right)=\frac{(\log x)^{r+1-\kappa}}{L^{(r+1-\kappa)}(0, \chi)}+O_{q}\left((\log x)^{r-\kappa}\right) \\
\quad+O\left((\log x)^{\omega\left(q^{\prime} / b^{\prime}\right)} \exp \left(C \sqrt{\omega\left(q^{\prime} / b^{\prime}\right) \log \left(q^{\prime} / b^{\prime}\right) \log \log x}\right)\right),
\end{aligned}
$$

where $C$ is a positive absolute constant, and $r$ indicates the number of the prime factors $p$ of $q$ with $\chi^{*}(p)=1$.

Proof. We separate the sum in three parts such that

$$
\begin{aligned}
& \sum_{|\eta|<T_{*}} \operatorname{Res}_{s=i \eta}\left(\frac{x^{s}}{L(s, \chi) s}\right)=\operatorname{Res}_{s=0}\left(\frac{x^{s}}{L(s, \chi) s}\right)+\sum_{0<|\eta| \leq T_{0}(q)} \operatorname{Res}_{s=i \eta}\left(\frac{x^{s}}{L(s, \chi) s}\right) \\
& +\sum_{T_{0}(q)<|\eta|<T_{*}} \operatorname{Res}_{s=i \eta}\left(\frac{x^{s}}{L(s, \chi) s}\right) .
\end{aligned}
$$

By estimates (6.3), (6.5), and (6.6), we can find a constant $T_{0}(q)$, depending only on $q$, with $T_{0}(q) \in[\omega(q)+5, \omega(q)+6]$ and

$$
\left|F_{q, \chi^{*}}\left(\sigma+i T_{0}(q)\right)\right|^{-1} \leq \exp \left(\frac{C(\log q)^{2}}{\log \log (q+5)}\right)
$$

for $|\sigma| \leq 2$. By the Leibniz rule, the first sum is estimated by

$$
\begin{aligned}
\operatorname{Res}_{s=0} & \left(\frac{x^{s}}{L(s, \chi) s}\right) \\
& =\frac{1}{(r+1-\kappa) !} \lim _{s \rightarrow 0} \sum_{j=0}^{r+1-\kappa}\left(\begin{array}{c}
r+1-\kappa \\
j
\end{array}\right) x^{s}(\log x)^{r+1-\kappa-j} \frac{\mathrm{d}^{j}}{\mathrm{~d}^{j}}\left(\frac{s^{r+2-\kappa}}{L(s, \chi) s}\right) \\
& =\frac{(\log x)^{r+1-\kappa}}{L^{(r+1-\kappa)}(0, \chi)}+O_{q}\left((\log x)^{r-\kappa}\right) .
\end{aligned}
$$


On the second sum, by the simplicity of zeros of $F_{q, \chi^{*}}$, we have

$$
\sum_{0<|\eta| \leq T_{0}(q)} \operatorname{Res}_{s=i \eta}\left(\frac{x^{s}}{L(s, \chi) s}\right)=\sum_{0<|\eta| \leq T_{0}(q)} \frac{x^{i \eta}}{L^{\prime}(i \eta, \chi) i \eta}=O_{q}(1) .
$$

As for the third sum, we use the result that

$$
\frac{1}{L(s, \chi)} \ll \log (q|t|)
$$

for

$$
\sigma \geq 1-\frac{c}{\log q+(\log (|t|))^{2 / 3}(\log \log (|t|))^{1 / 3}} \quad \text { and } \quad|t| \geq 5
$$

where $c$ is a positive absolute constant. On the region (7.5), we refer to $[11$, Section 9.5]. The author cannot find the above upper bound (7.4) in this region in references, but we can obtain it by the standard method (cf. [12, Section 11.1]). Here, we put $\varepsilon(x)=\frac{1}{\log x}$ with $x \geq$ $q^{C} \exp \left(C(\log T)^{2 / 3}(\log \log T)^{1 / 3}\right)$. Then, by the residue theorem, the third sum can be written as

$$
\begin{aligned}
& \sum_{T_{0}(q)<\eta<T_{*}} \operatorname{Res}_{s=i \eta}\left(\frac{x^{s}}{L(s, \chi) s}\right) \\
& =\frac{1}{2 \pi i}\left(\int_{\varepsilon(x)+i T_{0}(q)}^{\varepsilon(x)+i T_{*}}+\int_{\varepsilon(x)+i T_{*}}^{-\varepsilon(x)+i T_{*}}+\int_{-\varepsilon(x)+i T_{*}}^{-\varepsilon(x)+i T_{0}(q)}+\int_{-\varepsilon(x)+i T_{0}(q)}^{\varepsilon(x)+i T_{0}(q)}\right) \frac{x^{s}}{L(s, \chi) s} \mathrm{~d} s .
\end{aligned}
$$

From the definitions of $T_{0}(q)$ and $T_{*}$, we find that

$$
\left(\int_{\varepsilon(x)+i T_{*}}^{-\varepsilon(x)+i T_{*}}+\int_{-\varepsilon(x)+i T_{0}(q)}^{\varepsilon(x)+i T_{0}(q)}\right) \frac{x^{s}}{L(s, \chi) s} \mathrm{~d} s=O_{q}(1) .
$$

On the other hand, by using Lemma 9 and Proposition 6 with $h^{\prime}$ $=\sqrt{\frac{\omega\left(q^{\prime} / b^{\prime}\right)}{\log \left(q^{\prime} / b^{\prime}\right) \log \log x}}$, we have

$$
\begin{aligned}
& \left|F_{q, \chi^{*}}(\sigma+i t)\right| \\
& \quad \geq \exp \left(-C_{1} \sqrt{\omega\left(q^{\prime} / b^{\prime}\right) \log \left(q^{\prime} / b^{\prime}\right) \log \log x}\right) \prod_{|t-\eta| \leq h^{\prime}}|\sigma+i(t-\eta)| \\
& \quad \geq \exp \left(-C_{2} \sqrt{\omega\left(q^{\prime} / b^{\prime}\right) \log \left(q^{\prime} / b^{\prime}\right) \log \log x}\right)\left(\frac{1}{\log x}\right)^{\omega\left(q^{\prime} / b^{\prime}\right)}
\end{aligned}
$$


on the lines $|\sigma|=(\log x)^{-1},|t| \geq 2$. Now $b$ denotes the modulus of $\chi^{*}$. Therefore, for $|\sigma|=(\log x)^{-1},|t| \geq \frac{\log q}{\log \log (q+2))}+5$, we have

$$
\begin{aligned}
& \frac{1}{L(\sigma+i t, \chi)} \\
& \quad \ll \exp \left(C_{2} \sqrt{\omega\left(q^{\prime} / b^{\prime}\right) \log \left(q^{\prime} / b^{\prime}\right) \log \log x}\right) \frac{(\log x)^{\omega\left(q^{\prime} / b^{\prime}\right)}}{b|t|\left|L\left(1+\sigma+i t, \chi^{*}\right)\right|} \\
& \ll \exp \left(C_{2} \sqrt{\omega\left(q^{\prime} / b^{\prime}\right) \log \left(q^{\prime} / b^{\prime}\right) \log \log x}\right)(\log x)^{\omega\left(q^{\prime} / b^{\prime}\right) \frac{\log (b|t|)}{b|t|}}
\end{aligned}
$$

by Lemma 2 . Hence we have

$$
\begin{aligned}
& \int_{ \pm \varepsilon(x)+i T_{0}(q)}^{ \pm \varepsilon(x)+i T_{*}} \frac{x^{s}}{L(s, \chi) s} \mathrm{~d} s \\
& \quad \ll \exp \left(C_{2} \sqrt{\omega\left(q^{\prime} / b^{\prime}\right) \log \left(q^{\prime} / b^{\prime}\right) \log \log x}\right)(\log x)^{\omega\left(q^{\prime} / b^{\prime}\right)} \int_{T_{0}(q)}^{T_{*}} \frac{\log (b t)}{b t^{2}} \mathrm{~d} t \\
& \quad \ll \exp \left(C_{2} \sqrt{\omega\left(q^{\prime} / b^{\prime}\right) \log \left(q^{\prime} / b^{\prime}\right) \log \log x}\right)(\log x)^{\omega\left(q^{\prime} / b^{\prime}\right)} .
\end{aligned}
$$

Thus we have

$$
\begin{aligned}
& \sum_{T_{0}(q)<\eta<T_{*}} \operatorname{Res}_{s=i \eta}\left(\frac{x^{s}}{L(s, \chi) s}\right) \\
& \ll \exp \left(C \sqrt{\omega\left(q^{\prime} / b^{\prime}\right) \log \left(q^{\prime} / b^{\prime}\right) \log \log x}\right)(\log x)^{\omega\left(q^{\prime} / b^{\prime}\right)},
\end{aligned}
$$

where $C$ is a positive absolute constant. Similarly, we have

$$
\begin{aligned}
& \sum_{-T_{*}<\eta<-T_{0}(q)} \operatorname{Res}_{s=i \eta}\left(\frac{x^{s}}{L(s, \chi) s}\right) \\
& \ll \exp \left(C \sqrt{\omega\left(q^{\prime} / b^{\prime}\right) \log \left(q^{\prime} / b^{\prime}\right) \log \log x}\right)(\log x)^{\omega\left(q^{\prime} / b^{\prime}\right)} .
\end{aligned}
$$

From the above estimates, we obtain (7.3).

Proof of Theorem 3. Let $x \geq 3$. If $x \leq \exp \left(q^{1 / 3}\right)$, estimate (2.4) holds since the implicit constant may depend on $q$. Suppose that $x$ is half integer with $x \geq \exp \left(q^{1 / 3}\right)$. By Lemmas 10, 11, we have

$$
\begin{aligned}
\sum_{|\eta|<T_{*}} \operatorname{Res}_{s=i \eta}\left(L_{-1}(s ; q, a) \frac{x^{s}}{s}\right) & +\sum_{l=1}^{\infty} \operatorname{Res}_{s=-l}\left(L_{-1}(s ; q, a) \frac{x^{s}}{s}\right) \\
& \ll_{q}(\log x)^{\omega(q)} \exp (C \sqrt{\omega(q) \log (q) \log \log x}) .
\end{aligned}
$$


This estimate, Theorem 2 with $T=x$, and our assumptions of the generalized Riemann Hypothesis and multiplicities of zeros yield that

$$
\begin{aligned}
M^{*}(x ; q, a)=x^{1 / 2} \sum_{0<|\gamma|<T_{\nu}} \frac{x^{i \gamma}}{\rho} \lim _{s \rightarrow \rho}(s & -\rho) L_{-1}(s ; q, a) \\
& +\operatorname{Res}_{s=\frac{1}{2}}\left(L_{-1}(s ; q, a) \frac{x^{s}}{s}\right)+O_{\varepsilon}\left(x^{\varepsilon}\right)
\end{aligned}
$$

for some $T_{\nu} \in[x, 2 x]$.

Let $m(q)$ be the maximum in the multiplicities of the zeros of Dirichlet $L$-functions modulo $q$ at $s=\frac{1}{2}$. We then find that

$$
\operatorname{Res}_{s=\frac{1}{2}}\left(L_{-1}(s ; q, a) \frac{x^{s}}{s}\right) \ll_{q} x^{1 / 2}(\log x)^{m(q)-1} .
$$

By the definition of $L_{-1}(s ; q, a)$, we can write

$$
\begin{aligned}
\sum_{0<|\gamma|<T_{\nu}} \frac{x^{i \gamma}}{\rho} \lim _{s \rightarrow \rho}(s-\rho) L_{-1}(s ; q, a) & =\frac{1}{\varphi(q)} \sum_{\chi \bmod q} \bar{\chi}(a) \sum_{0<|\gamma|<T_{\nu}} \frac{x^{i \gamma}}{L^{\prime}(\rho, \chi) \rho} \\
& \ll \frac{1}{\varphi(q)} \sum_{\chi \bmod } \sum_{q<|\gamma| \leq 2 x} \frac{1}{\left|\gamma L^{\prime}(\rho, \chi)\right|} .
\end{aligned}
$$

Using estimate (2.3) with $\lambda=-\frac{1}{2}$ and partial summation, we find that

$$
\sum_{0<|\gamma| \leq 2 x} \frac{1}{\left|\gamma L^{\prime}(\rho, \chi)\right|}=\frac{J_{-1 / 2, \chi}(2 x)}{2 x}+\int_{\left|\gamma_{1}\right|}^{2 x} \frac{J_{-1 / 2, \chi}(\xi)}{2 \xi^{2}} \mathrm{~d} \xi \ll_{q}(\log x)^{5 / 4} .
$$

Here, $\gamma_{1}$ indicates the imaginary part of the nearest zero from the real axis. Thus, we obtain Theorem 3 .

\section{Proof of Theorem 6}

Proof of Theorem 6 . Let $\chi$ be a primitive Dirichlet character modulo $q$. Assume the simple zero conjecture for $L(s, \chi)$. Now, there exists the domain $-3 / 4 \leq \sigma \leq 2,0<t \leq 2 \delta(\chi)$ with $\delta(\chi) \leq 5$ such that this domain does not have zeros of $L(s, \chi)$ since $L(s, \chi)$ is entire function. In addition, by the compactness of the line segment $-3 / 4 \leq \sigma \leq 2, t=\delta$, and the continuity of $L(s, \chi)$, we have

$$
\frac{1}{|L(s, \chi)|} \leq C(\chi)
$$

on the same domain, where $C(\chi)$ is a sufficiently large constant depending only on $\chi$. 
Let $T \geq \exp \left(q^{1 / 3}\right)$. Here, by Proposition 1 and Lemma 2, there exist some $T_{\nu} \in[T, 2 T]$ satisfying

$$
\left|L\left(\sigma+i T_{\nu}, \chi\right)\right|^{-1} \leq \exp \left(C(\log \log T)^{2}\right)
$$

for $-1 \leq \sigma \leq 2$.

Now, by the residue theorem, we have

$$
\begin{aligned}
& \sum_{0<\gamma<T_{\nu}} \frac{1}{L^{\prime}(\rho, \chi)} \\
& \quad=\frac{1}{2 \pi i}\left(\int_{2+i \delta(\chi)}^{2+i T_{\nu}}+\int_{2+i T_{\nu}}^{-3 / 4+i T_{\nu}}+\int_{-3 / 4+i T_{\nu}}^{-3 / 4+i \delta(\chi)}+\int_{-3 / 4+i \delta(\chi)}^{2+i \delta(\chi)}\right) \frac{\mathrm{d} s}{L(s, \chi)} .
\end{aligned}
$$

From the way of taking $\delta(\chi)$ and $T_{\nu}$, the integrals on the horizontal line parts are estimated by

$$
\left|\int_{-3 / 4+i T_{\nu}}^{2 \pm i T_{\nu}} \frac{\mathrm{d} s}{L(s, \chi)}\right| \ll \exp \left(C(\log \log T)^{2}\right),
$$

and

$$
\left|\int_{-3 / 4+i \delta(\chi)}^{2+i \delta(\chi)} \frac{\mathrm{d} s}{L(s, \chi)}\right| \leq 3 C(\chi) .
$$

In addition, we have

$$
\left|\int_{-3 / 4+i \delta(\chi)}^{-3 / 4+i T_{\nu}} \frac{\mathrm{d} s}{L(s, \chi)}\right| \ll 1
$$

since $|L(-3 / 4+i t, \chi)|^{-1} \ll(|t|+1)^{-5 / 4}$ by Lemma 2 . On the first integral term, by the Dirichlet series expression, we have

$$
\begin{aligned}
\frac{1}{2 \pi i} \int_{2+i \delta(\chi)}^{2+i T_{\nu}} \frac{\mathrm{d} s}{L(s, \chi)} & =\frac{1}{2 \pi} \int_{\delta(\chi)}^{T_{\nu}} \sum_{n=1}^{\infty} \frac{\mu(n) \chi(n)}{n^{2+i t}} \mathrm{~d} t \\
& =\frac{T_{\nu}}{2 \pi}+\frac{1}{2 \pi} \sum_{n=2}^{\infty} \frac{\mu(n) \chi(n)}{n^{2}} \int_{\delta(\chi)}^{T_{\nu}} n^{-i t} \mathrm{~d} t+O(1) \\
& =\frac{T_{\nu}}{2 \pi}+O\left(\sum_{n=2}^{\infty} \frac{1}{n^{2} \log n}\right)=\frac{T_{\nu}}{2 \pi}+O(1) .
\end{aligned}
$$

Hence we have

$$
\sum_{0<\gamma<T_{\nu}} \frac{1}{L^{\prime}(\rho, \chi)}=\frac{T_{\nu}}{2 \pi}+O\left(\exp \left(C(\log \log T)^{2}\right)+C(\chi)\right) .
$$


In particular, for $T \geq T_{0}(q)$ with sufficiently large constant $T_{0}(q)$ depending only on $q$, we obtain

$$
\sum_{0<\gamma \leq 2 T} \frac{1}{\left|L^{\prime}(\rho, \chi)\right|} \geq \sum_{0<\gamma<T_{\nu}} \frac{1}{\left|L^{\prime}(\rho, \chi)\right|} \geq\left|\sum_{0<\gamma<T_{\nu}} \frac{1}{L^{\prime}(\rho, \chi)}\right| \gg T,
$$

which completes the proof of Theorem 6 .

\section{Results for number fields}

In this section, we describe the results for number fields. It is well known, by the Kronecker-Weber theorem, that if $K / \mathbb{Q}$ is an abelian extension of number field, then there exists a positive integer $q$ and a subset $X(K)$ of Dirichlet characters modulo $q$ such that

$$
\zeta_{K}(s)=\prod_{\chi \in X(K)} L\left(s, \chi^{*}\right),
$$

where $\chi^{*}$ is the primitive Dirichlet character inducing $\chi$. This formula is written in for example [14, Theorem 8.2].

The following corollary is an immediate consequence of Proposition 1 and equation (9.1).

Corollary 2. Let $\alpha \geq 13$ and $T \geq T_{0}(\alpha)>0$ with $T_{0}(\alpha)$ a sufficiently large constant depending only on $\alpha$. If $K$ is an Abelian number field, and $K_{m}$ is the smallest cyclotomic field satisfying $K \subset K_{m}$, we have

$$
\min _{T \leq t \leq 2 T}\left(\max _{\frac{1}{2} \leq \sigma \leq 2}\left|\zeta_{K}(\sigma+i t)\right|^{-1}\right) \leq \exp \left(C \alpha(\# X(K))(\log \log (m T))^{2}\right)
$$

for $m \leq(\log T)^{\alpha / 4}$, where $C$ is a positive absolute constant.

Remark 3. Corollary 2 holds for an Abelian number field. On the other hand, it is difficult to extend Corollary 2 to any number field. The reason is that a zero density theorem for Dirichlet $L$-functions in the region close to critical line plays an important role in the proof of Proposition 1, but it is difficult to obtain the zero density theorem of the same type for Dedekind zeta-functions.

By Corollary 2, we can obtain the explicit formula for the summatory function of the Möbius function $\mu_{K}$ on an Abelian number field $K$. This Möbius function $\mu_{K}$ is defined by

$$
\mu_{K}(\mathfrak{a})= \begin{cases}1 & \text { if } \mathfrak{a}=O_{K}, \\ (-1)^{k} & \text { if } \mathfrak{a} \text { is the product of } k \text { distinct prime ideals, } \\ 0 & \text { otherwise. }\end{cases}
$$


Here, we define the summatory function $M_{K}^{*}(x)$ by

$$
M_{K}^{*}(x)=\sum_{N(\mathfrak{a}) \leq x}^{\prime} \mu_{K}(\mathfrak{a})
$$

where the sum on the right hand side runs over integral ideals $\mathfrak{a}$ of the ring $O_{K}$, and $N(\mathfrak{a})$ is the absolute norm of $\mathfrak{a}$, and $\sum^{\prime}$ indicates that if $x$ is an integer, then $\sum_{N(\mathfrak{a}) \leq x}^{\prime}=\sum_{N(\mathfrak{a})<x}+\frac{1}{2} \sum_{N(\mathfrak{a})=x}$. Let $n_{x}$ denote one of the nearest positive integer from $x$ other than $x$ itself such that there exist ideals $\mathfrak{a} \subset O_{K}$ with $N(\mathfrak{a})=n_{x}$ and $\mu_{K}(\mathfrak{a}) \neq 0$. If there exist several such integers, then we understand that $n_{x}$ is the one for which $a_{n_{x}}$ is biggest. Here, $a_{n}$ means the number of ideals such that $N(\mathfrak{a})=n$. Then we obtain the following theorem.

Theorem 7. Let $K$ be an Abelian number field, $K_{m}$ be the smallest cyclotomic field satisfying $K \subset K_{m}, x>0, T \geq \max \left\{T_{0}, \exp \left(m^{1 / 3}\right), 2 / x\right\}$ with $T_{0}$ a sufficiently large absolute constant. Then, there exists some $T_{\nu} \in$ $[T, 2 T]$ such that

$$
\begin{aligned}
M_{K}^{*}(x)=\sum_{|\gamma|<T_{\nu}} \frac{1}{(m(\rho)-1) !} \lim _{s \rightarrow \rho} \frac{\mathrm{d}^{m(\rho)-1}}{\mathrm{~d} s^{m(\rho)-1}}\left((s-\rho)^{m(\rho)} \frac{x^{s}}{\zeta_{K}(s) s}\right) \\
+\sum_{l=0}^{\infty} \operatorname{Res}_{s=-l}\left(\frac{x^{s}}{\zeta_{K}(s) s}\right)+R^{\prime} .
\end{aligned}
$$

Here, $R^{\prime}$ satisfies the estimate

$$
\begin{aligned}
R^{\prime} \ll & \frac{x}{T} \min \left\{e^{n_{K} / x}(\log (x+2))^{n_{K}}, \kappa_{K} \log (x+2)+\frac{\Phi_{0}(K)}{(\log (x+2))^{-1}+1 / n_{K}}\right\} \\
& +\frac{x}{T} \exp \left(C n_{K}(\# X(K))(\log \log T)^{2}\right)+a_{n_{x}} \min \left\{1, \frac{x}{T\left|x-n_{x}\right|}\right\},
\end{aligned}
$$

where $n_{K}$ is the degree of $K, \kappa_{K}$ is the residue of $\zeta_{K}$ at $s=1$, and $\Phi_{0}(K)$ is a constant depending only on $K$.

Remark 4. The constant $\Phi_{0}(K)$ comes from the inequality

$$
\left|\sum_{N(\mathfrak{a}) \leq x} 1-\kappa_{K} x\right| \leq \Phi_{0}(K) x^{1-1 / n_{K}} .
$$

We do not consider refined upper bounds of $\Phi_{0}(K)$ in this paper, but it was studied by Murty and Order in [13].

Remark 5. We can remove the condition "Abelian" in this theorem by assuming the zero density estimate for Dedekind zeta-functions. 
Similarly to Theorem 3, we can apply a generalization of the GonekHejhal Conjecture to Theorem 7.

Conjecture 3 (The Generalized Gonek-Hejhal Conjecture for Dedekind zeta-functions). Assume that the all nontrivial zeros of Dedekind zetafunctions are simple except for the zero at $s=\frac{1}{2}$. For sufficiently large number $T>0$,

$$
J_{\lambda, K}(T):=\sum_{0<|\gamma| \leq T}\left|\zeta_{K}^{\prime}(\rho)\right|^{2 \lambda} \asymp_{\lambda, K} T(\log T)^{(\lambda+1)^{2}}
$$

holds for $\lambda>-\frac{3}{2}$.

Using this, we can obtain the following theorem.

Theorem 8. Let $K / \mathbb{Q}$ be a number field. Assume that the Riemann Hypothesis for $\zeta_{K}(s)$ is true, the all nontrivial zeros of $\zeta_{K}(s)$ are simple, and that $J_{-1 / 2, K}(T):=\sum_{0<|\gamma| \leq T}\left|\zeta_{K}^{\prime}(\rho)\right|^{-1} \ll_{K} T(\log T)^{1 / 4}$. Then, we have

$$
M_{K}^{*}(x) \ll_{K} x^{1 / 2}\left((\log x)^{5 / 4}+(\log x)^{m(K)-1}\right),
$$

where $m(K)$ is the multiplicity of the zero of the Dedekind zeta-function $\zeta_{K}(s)$ at $s=\frac{1}{2}$.

We can also obtain a theorem for $\zeta_{K}(s)$ with an Abelian number field $K$, similar to Theorem 6 . It is the following result.

Theorem 9. Let $K$ be an Abelian number field, $K_{m}$ be the smallest cyclotomic field satisfying $K \subset K_{m}$, and assume the simplicity of all complex zeros of $\zeta_{K}(s)$. Then, for $T>\exp \left(m^{1 / 3}\right)$, there exist some $T_{\nu} \in[T, 2 T]$ satisfying

$$
\sum_{0<\gamma<T_{\nu}} \frac{1}{\zeta_{K}^{\prime}(\rho)}=\frac{T_{\nu}}{2 \pi}+O\left(\exp \left(C(\# X(K))(\log \log T)^{2}\right)+C(K)\right),
$$

where $X(K)$ is the same as in equation (9.1), the sum on the left hand side runs over non-trivial zeros $\rho=\beta+i \gamma$ of $\zeta_{K}(s)$, and $C(K)$ is a sufficiently large constant depending only on $K$. In particular, for any $T \geq T_{0}(K)>0$ with a sufficiently large constant $T_{0}(K)$ depending only on $K$, we have

$$
\sum_{0<\gamma \leq T} \frac{1}{\left|\zeta_{K}^{\prime}(\rho)\right|} \gg T \text {. }
$$

We omit the proof of Theorem 9 because the proof is almost the same as the proof of Theorem 6 by using Corollary 2 instead of Proposition 1.

Similarly to the case of Dirichlet $L$-functions, estimate (9.6) is useful when we consider the exact behavior of some partial sum of some 
Möbius functions under the Linear Independence Conjecture. Actually, applying (9.6), we can show that

$$
\varlimsup_{x \rightarrow \infty} \frac{M_{K}^{*}(x)}{x^{1 / 2}}= \pm \infty
$$

for any Abelian number field $K$ under the following conjecture.

Conjecture (Linear Independence Conjecture for Dedekind zeta-functions (cf. [3])). The positive imaginary parts of the zeros of any Dedekind zetafunction are linearly independent over $\mathbb{Q}$.

The proof of (9.7) is also similar to the proof of Corollary 15.7 in [12].

\section{Proofs of Theorems 7,8}

Firstly, we prepare some lemmas.

Lemma 12. Let $K$ be any number field, $n_{K}$ be the degree of $K$, and $\kappa_{K}$ be the residue of $\zeta_{K}(s)$ at $s=1$. Then, for $\sigma>1$, we have

$$
\left|\zeta_{K}(\sigma+i t)\right| \leq \min \left\{\zeta(\sigma)^{n_{K}}, \frac{\sigma}{\sigma-1} \kappa_{K}+\frac{\sigma \Phi_{0}(K)}{\sigma-1+1 / n_{K}}\right\}=: \Phi_{K}(\sigma),
$$

where $\Phi_{0}(K)$ is the constant coming from inequality (9.4).

Proof. By the Euler product for Dedekind zeta-functions, we find that

$$
\begin{aligned}
\left|\zeta_{K}(\sigma+i t)\right| & \leq \prod_{\mathfrak{p}}\left(1-\frac{1}{N(\mathfrak{p})^{\sigma}}\right)^{-1}=\prod_{p} \prod_{\mathfrak{p} \mid p}\left(1-\frac{1}{p^{\operatorname{deg}(\mathfrak{p}) \sigma}}\right)^{-1} \\
& \leq \prod_{p}\left(1-\frac{1}{p^{\sigma}}\right)^{-n_{K}}=\zeta(\sigma)^{n_{K}} .
\end{aligned}
$$

On the other hand, using the partial summation and inequality (9.4), we have

$$
\begin{aligned}
\left|\zeta_{K}(\sigma+i t)\right| \leq \sigma \int_{1}^{\infty} \frac{A(x)}{x^{\sigma+1}} \mathrm{~d} x & \leq \sigma \int_{1}^{\infty} \frac{\kappa_{K} x+\Phi_{0}(K) x^{1-1 / n_{K}}}{x^{\sigma+1}} \mathrm{~d} \sigma \\
& =\frac{\sigma}{\sigma-1} \kappa_{K}+\frac{\sigma \Phi_{0}(K)}{\sigma-1+1 / n_{K}},
\end{aligned}
$$

where $A(x)=\sum_{N(\mathfrak{a}) \leq x} 1$. Hence we obtain Lemma 12 .

Lemma 13. Let $K$ be a number field. Let $d_{K}$ be the discriminant of $K$. If $|t| \geq 1$ and $\sigma \leq \frac{1}{2}$, then

$$
\begin{aligned}
\left|\zeta_{K}(s)\right| \gg \frac{1}{C^{n_{K}}}\left(\frac{(2 \pi e)^{n_{K}}}{\left|d_{K}\right||s|^{n_{K}}}\right)^{\sigma} & \left(\left|d_{K}\right||s|^{n_{K}}\right)^{1 / 2} \\
& \times \exp \left(n_{K}|t| \tan ^{-1}\left(\frac{1-\sigma}{|t|}\right)\right)\left|\zeta_{K}(1-s)\right| .
\end{aligned}
$$


If $|t| \leq 1, \sigma=-(r+1 / 2)$ with $r$ a positive integer, then

$$
\left|\zeta_{K}(s)\right| \gg \frac{1}{C^{n_{K}}}\left(\frac{(2 \pi e)^{n_{K}}}{\left|d_{K}\right||s|^{n_{K}}}\right)^{\sigma}\left(\left|d_{K}\right||\sigma|^{n_{K}}\right)^{1 / 2}\left|\zeta_{K}(1-s)\right| .
$$

The above $C$ is an absolute constant.

Proof. By the functional equation for $\zeta_{K}(s)$ which is

$$
\begin{aligned}
\zeta_{K}(s)=2^{n_{K}}(2 \pi)^{n_{K}(s-1)}\left|d_{K}\right|^{1 / 2-} & (\Gamma(1-s))^{n_{K}} \\
& \times\left(\sin \frac{\pi s}{2}\right)^{r_{1}+r_{2}}\left(\cos \frac{\pi s}{2}\right)^{r_{2}} \zeta_{K}(1-s),
\end{aligned}
$$

and the Stirling formula, we obtain this lemma.

Proof of Theorem \%. Let $x>0, T \geq \max \left\{9, \exp \left(m^{1 / 3}\right), 2 / x\right\}$ and $\sigma_{0}=$ $1+1 / \log (x+3)$. First, using Perron's formula, we have

$$
M_{K}^{*}(x)=\frac{1}{2 \pi i} \int_{\sigma_{0}-i T_{\nu}}^{\sigma_{0}+i T_{\nu}} \frac{x^{s}}{\zeta_{K}(s) s} \mathrm{~d} s+O\left(\frac{x}{T} \Phi_{K}\left(\sigma_{0}\right)+\min \left\{1, \frac{x}{T\langle x\rangle}\right\}\right),
$$

where $T_{\nu}$ satisfies the inequality

$$
\left|\zeta_{K}\left(\sigma+i T_{\nu}\right)\right|^{-1} \leq \exp \left(C n_{K}(\# X(K))(\log \log T)^{2}\right)
$$

for any $-1 \leq \sigma \leq 2$ with $T_{\nu} \in[T, 2 T]$ by Corollary 2 and Lemma 13 . Here we remark that $T \geq \exp \left(m^{1 / 3}\right) \gg m$.

Let $R=r+\frac{1}{2}$ with positive integer $r$ satisfying $r>T$. By the residue theorem, we have

$$
\begin{aligned}
& \frac{1}{2 \pi i} \int_{\sigma_{0}-i T_{\nu}}^{\sigma_{0}+i T_{\nu}} \frac{x^{s}}{\zeta_{K}(s) s} \mathrm{~d} s \frac{1}{2 \pi i}\left(\int_{-R+i T}^{\sigma_{0}+i T_{\nu}}+\int_{-R-i T_{\nu}}^{-R+i T_{\nu}}+\int_{\sigma_{0}-i T_{\nu}}^{-R-i T_{\nu}}\right) \frac{x^{s}}{\zeta_{K}(s) s} \mathrm{~d} s \\
& \quad+\sum_{|\gamma|<T_{*}} \operatorname{Res}_{s=\rho}\left(\frac{x^{s}}{\zeta_{K}(s) s}\right)+\sum_{0 \leq l<R} \operatorname{Res}_{s=-l}\left(\frac{x^{s}}{\zeta_{K}(s) s}\right) \\
&=: J_{1}+J_{2}+J_{3}+\sum_{|\gamma|<T_{\nu}} \operatorname{Res}_{s=\rho}\left(\frac{x^{s}}{\zeta_{K}(s) s}\right)+\sum_{0 \leq l<R} \operatorname{Res}_{s=-l}\left(\frac{x^{s}}{\zeta_{K}(s) s}\right) .
\end{aligned}
$$

Here, by the basic formula for residues, we find that

$$
\operatorname{Res}_{s=\rho}\left(\frac{x^{s}}{\zeta_{K}(s) s}\right)=\frac{1}{(m(\rho)-1) !} \lim _{s \rightarrow \rho} \frac{\mathrm{d}^{m(\rho)-1}}{\mathrm{~d} s^{m(\rho)-1}}\left((s-\rho)^{m(\rho)} \frac{x^{s}}{\zeta_{K}(s) s}\right) .
$$

Hence, we have (9.2) 
Next, we show (9.3). By Lemma $13, J_{2}$ is evaluated by

$$
\begin{aligned}
\left|J_{2}\right| & =\left|\int_{|t| \leq T_{\nu}} \frac{x^{-R+i t}}{\zeta_{K}(-R+i t)(-R+i t)} \mathrm{d} t\right| \\
& \ll x^{-R} C^{n_{K}} \int_{|t| \leq T_{\nu}}\left(\frac{(2 \pi e)^{n_{K}}}{R^{n_{K}}}\right)^{R} R^{-n_{K} / 2-1} \mathrm{~d} t \\
& \ll C^{n_{K}}\left(\frac{x}{(2 \pi e)^{n_{K}}}\right)^{-R} R^{-(R+1 / 2) n_{K}-1} T .
\end{aligned}
$$

Therefore, we have

$$
\lim _{R \rightarrow \infty} J_{2}=0 .
$$

Next, we estimate $J_{1}$. Now, we put

$$
J_{1}=\frac{1}{2 \pi i}\left(\int_{-1+i T_{\nu}}^{\sigma_{0}+i T_{\nu}}+\int_{-R+i T_{\nu}}^{-1+i T_{\nu}}\right) \frac{x^{s}}{\zeta_{K}(s) s} \mathrm{~d} s=: J_{1}^{\prime}+J_{1}^{\prime \prime} .
$$

By Lemma 12, Lemma 13 and estimate (10.3), we find that

$$
\begin{aligned}
\left|J_{1}^{\prime}\right| & \ll \int_{-1}^{\sigma_{0}} x T_{\nu}^{-1} \exp \left(C n_{K}(\# X(K))(\log \log T)^{2}\right) \mathrm{d} \sigma \\
& \ll \frac{x \exp \left(C n_{K}(\# X(K))(\log \log T)^{2}\right)}{T},
\end{aligned}
$$

and that

$$
\left|J_{1}^{\prime \prime}\right| \ll \frac{C^{n_{K}}}{T^{3 / 2}} \int_{-R}^{-1}\left(x T_{\nu}\right)^{\sigma} \mathrm{d} \sigma \ll \frac{C^{n_{K}}(x T)^{-1}}{T^{3 / 2} \log (x T)} .
$$

Hence we have

$$
J_{1} \ll \frac{x \exp \left(C n_{K}(\# X(K))(\log \log T)^{2}\right)}{T} .
$$

Similarly, by the Schwarz reflection principle, we have

$$
J_{3} \ll \frac{x \exp \left(C n_{K}(\# X(K))(\log \log T)^{2}\right)}{T} .
$$

Thus we obtain estimate (9.3), and complete the proof of Theorem 7 .

Next, we show Theorem 8. To do it, we evaluate the contribution from residues of trivial zeros. Let $r_{1}=r_{1}(K)$ be the number of real embeddings of $K$, and let $2 r_{2}=2 r_{2}(K)$ be the number of imaginary embeddings of $K$.

Lemma 14. Let $K$ be a number field. We have

$$
\sum_{l=0}^{\infty} \operatorname{Res}_{s=-l}\left(\frac{x^{s}}{\zeta_{K}(s) s}\right) \sim-\frac{2^{r_{1}+r_{2}} \pi^{r_{2}}(\log x)^{r_{1}+r_{2}-1}}{\left|d_{K}\right|^{1 / 2} \kappa_{K}}
$$

as $x \rightarrow+\infty$. 
Proof. We put $r=r_{1}+r_{2}-1$. Then, by the Leibniz rule, we find that

$$
\begin{aligned}
& \operatorname{Res}_{s=0}\left(\frac{x^{s}}{\zeta_{K}(s) s}\right)=\frac{1}{r !} \lim _{s \rightarrow 0} \frac{\mathrm{d}^{r}}{\mathrm{~d} s^{r}}\left(\frac{s^{r+1}}{\zeta_{K}(s) s} x^{s}\right) \\
& =\frac{1}{r !}(\log x)^{r} \lim _{s \rightarrow 0} \frac{s^{r}}{\zeta_{K}(s)}+\frac{\left(1-\delta_{0, r}\right)}{r !} \sum_{j=1}^{r}\left(\begin{array}{c}
r \\
j
\end{array}\right)(\log x)^{r-j} \lim _{s \rightarrow 0} \frac{\mathrm{d}^{j}}{\mathrm{~d} s^{j}}\left(\frac{s^{r}}{\zeta_{K}(s)}\right) \\
& =-\frac{(2 \pi)^{n_{K}}(\log x)^{r}(2 / \pi)^{r_{1}+r_{2}}}{2^{n_{K}}\left|d_{K}\right|^{1 / 2} \kappa_{K}}+O_{K}\left(\left(1-\delta_{0, r}\right)|\log x|^{r-1}\right) .
\end{aligned}
$$

On the other hand, for $l \geq 1$, we have

$$
\begin{aligned}
\operatorname{Res}_{s=-l}\left(\frac{x^{s}}{\zeta_{K}(s) s}\right) & =\frac{1}{2 \pi i} \int_{|s+l|=\frac{1}{\log (x+3)}} \frac{x^{s}}{\zeta_{K}(s) s} \mathrm{~d} s \\
& =\frac{1}{2 \pi \log (x+3)} \int_{0}^{2 \pi} \frac{x^{-l+\frac{e^{i \theta}}{\log (x+3)}} e^{i \theta}}{\zeta_{K}\left(-l+\frac{e^{i \theta}}{\log (x+3)}\right)\left(-l+\frac{e^{i \theta}}{\log (x+3)}\right)} \mathrm{d} \theta \\
& \ll \frac{x^{-l}}{l \log (x+3)} \int_{0}^{2 \pi} \frac{\mathrm{d} \theta}{\left|\zeta_{K}\left(-l+\frac{e^{i \theta}}{\log (x+3)}\right)\right|}
\end{aligned}
$$

by the Cauchy formula. Now, by the functional equation and the Stirling formula, we can find that if $l$ is even, then

$$
\left|\zeta_{K}\left(-l+\frac{e^{i \theta}}{\log (x+3)}\right)\right|^{-1} \ll C^{n_{K}}\left(\frac{2 \pi e}{l}\right)^{n_{K} l} l^{-n_{K} / 2}(\log (x+3))^{r_{1}+r_{2}},
$$

and that if $l$ is odd, then

$$
\left|\zeta_{K}\left(-l+\frac{e^{i \theta}}{\log (x+3)}\right)\right|^{-1} \ll C^{n_{K}}\left(\frac{2 \pi e}{l}\right)^{n_{K} l} l^{-n_{K} / 2}(\log (x+3))^{r_{2}} .
$$

Hence, for $l \geq 1$, we obtain

$$
\begin{aligned}
& \underset{s=-l}{\operatorname{Res}}\left(\frac{x^{s}}{\zeta_{K}(s) s}\right) \\
& \ll \begin{cases}\frac{C^{n_{K}} x^{-l}}{l^{n_{K} / 2+1}}\left(\frac{2 \pi e}{l}\right)^{n_{K} l}(\log (x+3))^{r_{1}+r_{2}-1} & \text { if } l \text { is even, } \\
\left(1-\delta_{0, r_{2}}\right) \frac{C^{n_{K}} x^{-l}}{l^{n_{K} / 2+1}}\left(\frac{2 \pi e}{l}\right)^{n_{K} l}(\log (x+3))^{r_{2}-1} & \text { if } l \text { is odd. }\end{cases}
\end{aligned}
$$

From the above estimates, we obtain this lemma.

Proof of Theorem 8. Note that we can prove Theorem 7 for $\zeta_{K}(s)$ under the Riemann Hypothesis for $\zeta_{K}(s)$. Hence, assuming the Riemann Hypothesis 
and simplicity for nontrivial zeros and using Theorem 7 with $T=x$ and Lemma 14, we have

$$
M_{K}^{*}(x)=x^{1 / 2} \sum_{0<|\gamma|<T_{\nu}} \frac{x^{i \gamma}}{\zeta_{K}^{\prime}(\rho) \rho}+\operatorname{Res}_{s=\frac{1}{2}}\left(\frac{x^{s}}{\zeta_{K}(s) s}\right)+O_{K, \varepsilon}\left(x^{\varepsilon}\right)
$$

for any $\varepsilon>0$ and for some $T_{\nu} \in[x, 2 x]$. Evaluating the contribution from nontrivial zeros and the residue at $s=\frac{1}{2}$ similarly to the proof of Theorem 3 , we can obtain Theorem 8 .

\section{References}

[1] K. M. BARTZ, "On some complex explicit formulae connected with the Möbius function. II", Acta Arith. 57 (1991), no. 4, p. 295-305.

[2] G. Bhowmik, K. Halupczok, K. мatsumoto \& Y. Suzuki, "Goldbach representations in arithmetic progressions and zeros of Dirichlet L-functions", Mathematika 65 (2019), no. 1, p. $57-97$.

[3] J. B. Conrey, "The Riemann hypothesis", Notices Am. Math. Soc. 50 (2003), no. 3, p. 341353.

[4] M. Z. Garaev \& A. Sankaranarayanan, "The sum involving derivative of $\zeta(s)$ over simple zeros", J. Number Theory 117 (2006), no. 1, p. 122-130.

[5] S. M. Gonek, "On negative moments of the Riemann zeta-function", Mathematika 36 (1989), no. 1, p. 71-88.

[6] — , "An explicit formula of Landau and its applications to the theory of the zetafunction", in A tribute to Emil Grosswald: number theory and related analysis, Contemporary Mathematics, vol. 143, Springer, 1993, p. 395-413.

[7] D. A. Hejhal, "On the distribution of $\log \left|\zeta^{\prime}\left(\frac{1}{2}+i t\right)\right|$ ", in Number theory, trace formulas, and discrete groups, Academic Press Inc., 1989, p. 343-370.

[8] A. E. Ingham, "On two conjectures in the theory of numbers", Am. J. Math. 64 (1942), p. 313-319.

[9] P. Kühn, N. Robles \& A. Roy, "On a class of functions that satisfies explicit formulae involving the Möbius function", Ramanujan J. 38 (2015), no. 2, p. 383-422.

[10] H. L. Montgomery, "Zeros of L-Functions", Invent. Math. 8 (1969), p. 346-354.

[11] - Ten lectures on the interface between analytic number theory and harmonic analysis, CBMS Regional Conference Series in Mathematics, vol. 84, American Mathematical Society, 1994.

[12] H. L. Montgomery \& R. C. Vaughan, Multiplicative Number Theory I. Classical Theory, Cambridge Studies in Advanced Mathematics, vol. 97, Cambridge University Press, 2007.

[13] M. R. Murty \& J. VAn Order, "Counting integral ideals in a number field", Expo. Math. 25 (2007), no. 1, p. 53-66.

[14] W. NArkiewicz, Elementary and Analytic Theory of Algebraic Numbers, 2nd ed., Springer, 1990, substantially revised and extended.

[15] N. NG, "The distribution of the summatory function of the Möbius function", Proc. Lond. Math. Soc. 89 (2004), p. 361-389.

[16] K. Ramachandra \& A. Sankaranarayanan, "Notes on the Riemann zeta-function", $J$. Indian Math. Soc., New Ser. 57 (1991), no. 1-4, p. 67-77.

[17] K. Soundararajan, "Partial sums of the Möbius function", J. Reine Angew. Math. 631 (2009), p. 141-152.

[18] E. C. Titchmarsh, The theory of the Riemann zeta-Function, 2nd ed., Oxford University Press, 1986, revised and with a preface by D. R. Heath-Brown.

[19] L. YE, "Bounding sums of the Möbius function over arithmetic progressions", https:// arxiv.org/abs/1406.7326, to appear in Acta. Arith., 2014. 
Shōta InOue

Department of Mathematics

Tokyo Institute of Technology

2-12-1 Ookayama, Meguro-ku

Tokyo 152-8551 Japan

E-mail: inoue.s.bd@m.titech.ac.jp 